\title{
A hybrid Machine Learning and Network Analysis approach reveals two Parkinson's Disease subtypes from 115 RNA-Seq post-mortem brain samples
}

\author{
Andrea Termine ${ }^{1, \dagger}$, Carlo Fabrizio ${ }^{1,+}$, Claudia Strafella ${ }^{2}$, Valerio Caputo ${ }^{2,3}$, Laura Petrosini $^{4}$, Carlo Caltagirone $^{5}$, \\ Raffaella Cascella $a^{3,6}$ and Emiliano Giardina ${ }^{2,7, *}$
}

1 Data Science Unit, IRCCS Santa Lucia Foundation c/o CERC; 00143 Rome, Italy; a.termine@hsantalucia.it (A.T.); c.fabrizio@hsantalucia.it (C.F.)

2 Genomic Medicine Laboratory UILDM, IRCCS Santa Lucia Foundation; 00179 Rome, Italy; claudia.strafella@gmail.com (C.S.); v.caputo91@gmail.com (V.C.); emiliano.giardina@uniroma2.it (E.G.)

3 Medical Genetics Laboratory, Department of Biomedicine and Prevention, Tor Vergata University; 00133 Rome, Italy; raffaella.cascella@gmail.com (R.C.)

4 Experimental and Behavioral Neurophysiology, IRCCS Santa Lucia Foundation c/o CERC; 00143 Rome, Italy; laura.petrosini@uniroma1.it

5 Department of Clinical and Behavioral Neurology, IRCCS Santa Lucia Foundation; 00179 Rome, Italy; c.caltagirone@hsantalucia.it

6 Department of Biomedical Sciences, Catholic University Our Lady of Good Counsel, 1000 Tirana, Albania.

7 UILDM Lazio ONLUS Foundation, Department of Biomedicine and Prevention, Tor Vergata University, 00133 Rome, Italy.

* Correspondence: emiliano.giardina@uniroma2.it

+ These authors contributed equally to this work.

\begin{abstract}
Precision medicine emphasizes fine-grained diagnostics, taking individual variability into account to enhance treatment effectiveness. Parkinson's Disease (PD) heterogeneity among individuals is a proof that disease subtypes exist, and assigning individuals to subgroups is necessary for a better understanding of disease mechanisms and designing precise treatment approaches. The purpose of this study was to identify PD subtypes using RNA-Seq data in a combined pipeline including unsupervised machine learning, bioinformatics, and network analysis. 210 post mortem brain RNA-Seq samples from PD $(n=115)$ and Normal Controls $(\mathrm{NC}, \mathrm{n}=95)$ were obtained with a systematic data retrieval following PRISMA statements and a fully data-driven clustering pipeline was performed to identify PD subtypes. Bioinformatics and Network analyses were performed to characterize the disease mechanisms of the identified PD subtypes and to identify target genes for drug repurposing. Two PD clusters were identified and 42 DEGs were found (p.adjusted $\leq 0.01$ ). PD clusters had significantly different gene network structures $(p<0.0001)$ and phenotype-specific disease mechanisms, highlighting the differential involvement of the $\mathrm{Wnt} / \beta$-catenin pathway regulating adult neurogenesis. NEUROD1 was identified as a key regulator of gene networks and ISX9 and PD98059 were identified as NEUROD1-interacting compounds with disease-modifying potential, reducing the effects of dopaminergic neurodegeneration. This hybrid data analysis approach could enable precision medicine applications by providing insights for the identification and characterization of pathological subtypes. This workflow has proven useful on PD brain RNA-Seq, but its application to other neurodegenerative diseases is encouraged.
\end{abstract}

Keywords: Data Science; Genomic Data Science; Machine Learning; Network Analysis; RNA-Seq; Precision Medicine; Subtyping; Parkinson's Disease;

\section{Introduction}

Parkinson's disease (PD) is the most common age-related motor neurodegenerative disease, affecting more than 6 million people worldwide, with rising incidence and prevalence imposing a mounting socioeconomic burden on society [1-3] and currently, no 
disease-modifying treatments are available [4-6]. The genetic basis of PD has been evaluated with several Genome-Wide Association Studies (GWAS), finally reporting up to 90 PD-associated risk variants in various cohorts [7-10]. However, genetic variants account for $\sim 20 \%$ of PD familial cases, while the etiology of most idiopathic cases is largely unclear as multiple genes and environmental factors are believed to be involved in idiopathic PD onset and progression [7]. Consequently, PD is heterogeneous in both clinical manifestations and progression, which serves as evidence for the existence of disease subtypes $[11,12]$. Defining which PD subtype we are facing is crucial to better understand underlying mechanisms, predict disease course, and eventually design personalized management strategies able to fully consider the genetic or other specific biological features that can be employed in a precision medicine approach addressed to match the patients' needs [11,13-15].

Empirical clustering stratifies patients based on demographic factors, clinical parameters, and genetic factors, making use of expert-based a priori conceptions. Up to now, these applications have shown limited sensitivity in detecting clinically useful classes of PD patients, thus hindering the development and deployment of better suited treatments [16]. It has been shown that the use of a priori assumptions in stratifying patients with complex diseases like PD can be appealing due to its simplicity, nevertheless this method has an arbitrary nature [16]. As an example, PD patients younger than 50 years of age represent only $5-10 \%$ of the total population [17] and thus they are simple to subset, whereas the later onset subtype $(\sim 90 \%)$ remains highly heterogeneous in its clinical presentation, and a clear boundary between groups is not easily achieved. The unreliability of this subtyping method became apparent as many studies used arbitrary cut-offs of 50,55 , and $60[16,18]$. Similarly, various authors have defined the motor subtypes of PD differently, providing ambiguous results for patients stratification, which indicates the arbitrary nature of a priori conceptions [16]. As a fine-grained diagnosis is pivotal for precision medicine applications, more sensitive methods are required. To this extent, datadriven clustering based on unsupervised Machine Learning (ML) could offer better results by applying unbiased statistical methods and a hypothesis-free approach. In fact, clustering algorithms aim at finding patterns within data distribution to define clusters, free from a priori assumptions about disease and patients. Data-driven subtyping applications on complex diseases are shaping our knowledge about the best suited treatments for patients with a fine-grained diagnostic definition, such as molecular subtypes of Alzheimer's Disease or colorectal cancer $[19,20]$. Coupling data-driven clustering methods with Next Generation Sequencing (NGS) is advancing biomedical research, and transcriptomics data from RNA-Seq can be used in Gene-Network analysis to identify gene co-expression/coregulation patterns, refining our understanding of complex biological systems. Datadriven PD subtyping has so far provided heterogeneous results due to disparate data sources and methods of clustering, which hampers understanding of idiopathic PD subgrouping [16]. In fact, even if data-driven clustering is a hypothesis-free approach, its results are dependent on the choice of the number of clusters and the used clustering technique. A fully data-driven clustering pipeline would address these limitations, providing more detailed diagnoses to facilitate precision medicine applications.

To the best of our knowledge, there are no published attempts at molecular subtyping of PD. Transcriptomic profiling from RNA-Seq data provides an in-depth characterization of complex diseases affecting the brain, reflecting the heterogeneity in the biological profiles of such pathologies. Moreover, network biology has not been extensively applied to PD research due to the lack of available data, thus information about gene interactions and regulations in PD is still poor [21]. Also, there are no available disease-modifying treatments for PD. Disease heterogeneity can be tidied up by coupling unsupervised ML methods with bioinformatics and network analyses on transcriptomic profiles. Regulatory networks identification would highlight genes acting as key regulators in each subtype, that can be used in a drug repurposing pipeline to foster tailored treatments definition. Here we show the implementation of a fully data-driven clustering pipeline and its application to PD subtyping. Our proposed novel methodology for better detailing 
diagnosis and accelerating drug repurposing could provide new research insights for assessing the efficacy of treatments. Our hybrid data-driven workflow is applied to gene expression data from idiopathic PD post-mortem brain samples from Gene Expression Omnibus (GEO) and PubMed databases. We aim to demonstrate that coupling unsupervised ML for disease subtyping with bioinformatics and Network Analysis for regulatory networks identification can clarify the molecular landscape of disease subtypes. The workflow integrates multiple unsupervised ML algorithms for disease subtyping, with independent clusterability assessment and best number of clusters determination. The clustering pipeline aims to optimize the internal clustering validation measures. Network and bioinformatics analyses have been used to isolate and characterize regulatory genes in common and specific disease networks. The obtained key genes from this workflow were tested in a drug repurposing pipeline, enabling us to propose a set of compounds with disease-modifying potential.

\section{Results}

\subsection{Clustering results and clinical characterization}

Two subtypes of PD, namely PD Cluster 1 (PDC1, n = 50) and PD Cluster 2 (PDC2, n $=65$ ) were identified through the cluster analysis pipeline on gene-expression data. Hierarchical K-means implementing centroid method with Manhattan distance was selected as the best clustering algorithm based on validation metrics (Silhouetteavg $=0.40$; Dunn = 0.05). The differences between PDC1 and PDC2 clinical and demographic variables were assessed. PDC2 lives longer than PDC1 (Wilcoxon Test p.value $=0.03$ ), and lower Braak scores than PDC1 were found (Fisher Test p.value $=0.004$ ), while no sex differences in the composition of the clusters were found (Fisher Test p.value $=0.21$ ) (Figure 1).
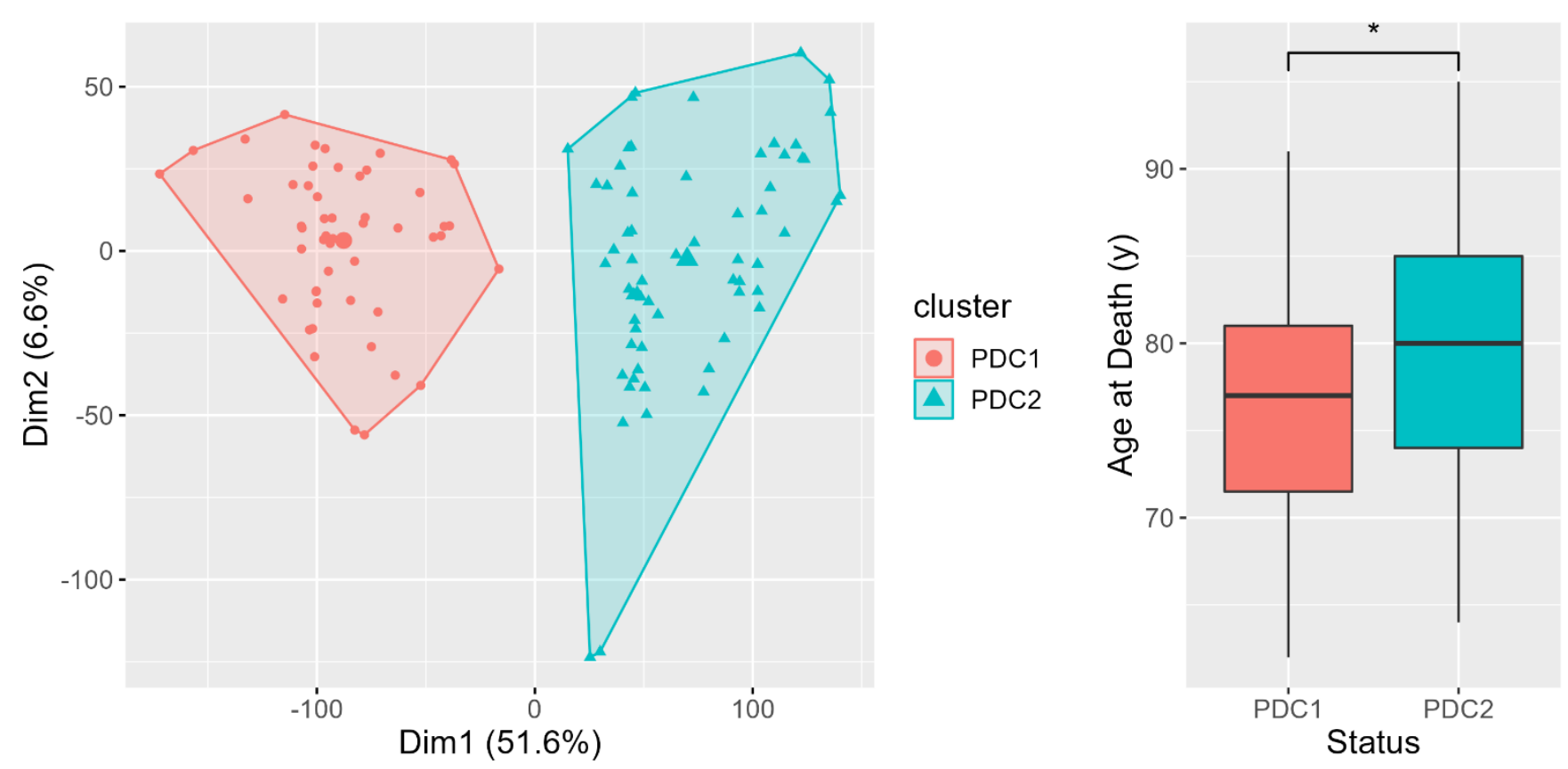

Figure 1. Clustered subjects and clinical characterization. (A) PCA plot for RNA-Seq data, clearly showing PDC1 and PDC2 separation. (B) Boxplot for PDC1 and PDC2 Age at Death, reporting a significant difference $\left({ }^{*} \mathrm{p} \leq 0.05 ;{ }^{* *} \mathrm{p} \leq 0.01 ;{ }^{* *} \mathrm{p} \leq 0.001\right)$. 
To investigate the genetic background regulating the differences in the survival rate of the two PD clusters we performed a differential gene expression analysis to retrieve DEGs. A comparison between the gene expression profiles of PDC1 and PDC2 was performed, yielding 42 DEGs with p.adjusted $\leq 0.01$ (Figure 2A). Among them, 6 Transcription Factors (TFs) were identified, mostly regulating the $\mathrm{Wnt} / \beta$-catenin pathway including TBR1, the basic helix-loop-helix (bHLH) TFs members NEUROD1, NEUROD2, NEUROD6, coupled with CBP/p300 modulators (CITED1, eEF1B $\gamma$ ). Notably, only $6 \%$ of the DEGs were recognized as PD-associated genes by GDA analysis. Functional enrichment analysis revealed that DEGs were mostly enriched in protein complexes associated with CBP/p300 modulation in the Wnt/ $\beta$-catenin pathway, namely IRF3-CBP (CORUM:299 p.adjusted < 0.01), BETA2-Cyclin D1 (CORUM:2635 p.adjusted < 0.01), SNW1 (CORUM:298 p.adjusted < 0.05), and VEGF (CORUM:298 p.adjusted < 0.05) complexes. Regulation of drug responses (GO:2001023 p.adjusted $<0.05$ ) was also altered between PDC1 and PDC2, with ADIRF and GRM2 overexpressed in PDC2. Overall, the obtained DEGs differently modulated the regulation of synapse structure or activity (GO:0050803 p.adjusted < 0.05; GO:0006865 p.adjusted $<0.05$ ), including alterations in the glutamatergic synapse due to mGluR2-mGluR4 complex (CORUM:6363 p.adjusted <0.01) modulation.

\subsection{PDC1 and PDC2 networks differ for neuroprotective pathways}

A network analysis approach was used to investigate differences in gene co-expression and connectivity between the two PD clusters. Two networks were obtained using DEGs to define nodes and PCIT values to define edges between nodes. Next, the label propagation algorithm was applied on the two networks to detect communities and 2 were found in PDC1 (Figure 2B), while no distinct communities were found in PDC2 (Figure $2 \mathrm{C}$ ). These structural differences were confirmed by the NCT test, showing that the subjects represented different PD subpopulations (p.value $<0.0001$ ). Communities in PDC1 were studied to identify the functional pathways enriched by their genes. Text-mining on STRINGdb showed that PDC1 community 1 (PPI score p.value $<0.0001$ ) was composed of DEGs belonging to Wnt/ $\beta$-catenin and TGF- $\beta /$ SMAD pathways, regulating adult neurogenesis [22], and functional enrichment analysis showed that DEGs in PDC1 community 1 significantly altered the Nuclear Factor of Activated T-cells 1 (NFATc1) transcription, which is a key regulator in Wnt signaling (TF:M04053, p.adjusted $<0.001$ ). To further explore functional alterations in PDC1 vs. PDC2 we decided to compare the intensity of the connections between nodes in the two networks. Although the overall level of connectivity is not different across PD subtypes (NCT, $\mathrm{S}=34.64, \mathrm{p}=0.08$ ), we observed 271 edges displaying different levels of strength between clusters (p.adjusted <0.01) (Figure 3A). Nodes in this differential network enriched the Neuronal helix-loop-helix TFs protein domain (PF12533, p.adjusted $<0.01$ ), which is required for neurogenesis. 
A

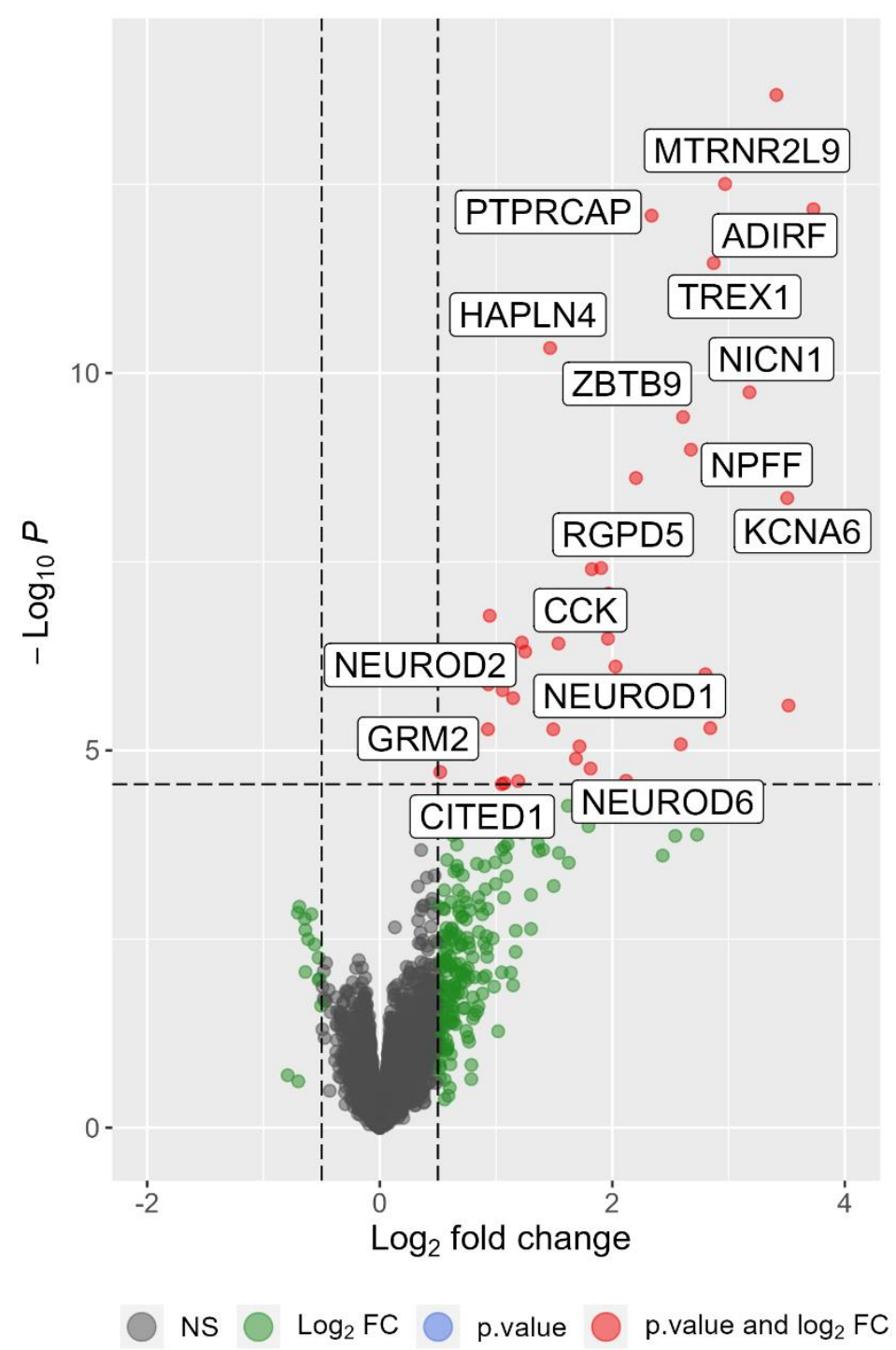

B

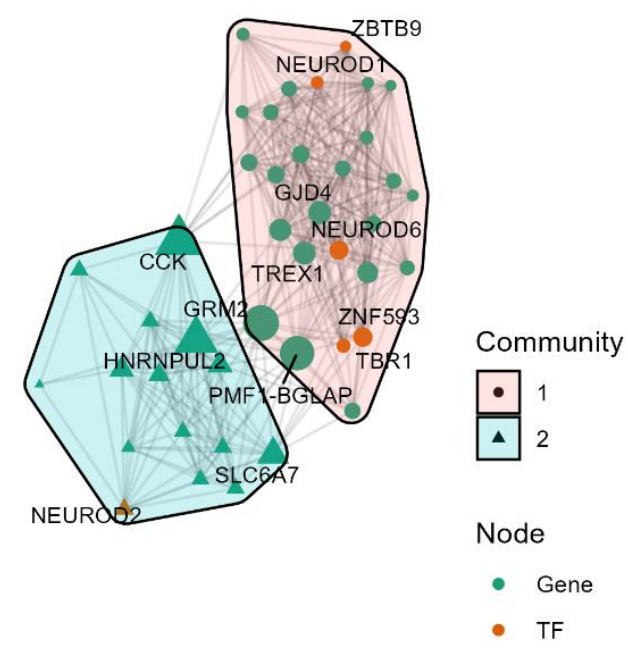

C

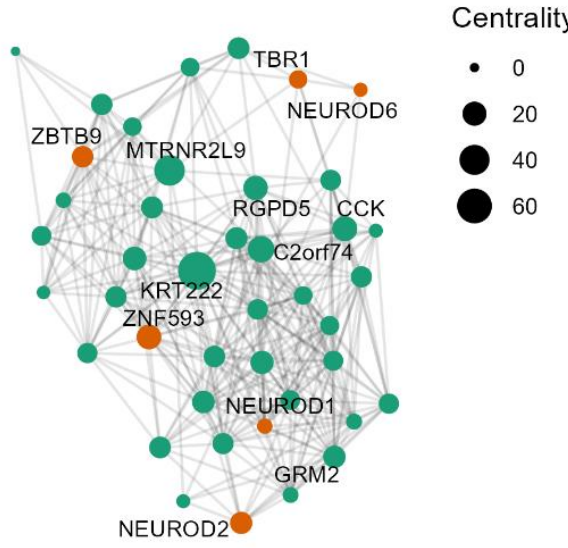

Figure 2. Differences between PDC1 and PDC2 gene expression. (A) Volcano plot reporting differences in gene expression where red points were DEGs. (B) Gene network for PDC1 showing nodes are segregated into 2 communities. (C) Gene network for PDC2, showing no segregation in communities. Both networks were built with the Kamada-Kawai layout, where each node is a gene and each edge is a PCIT value between genes. 
A

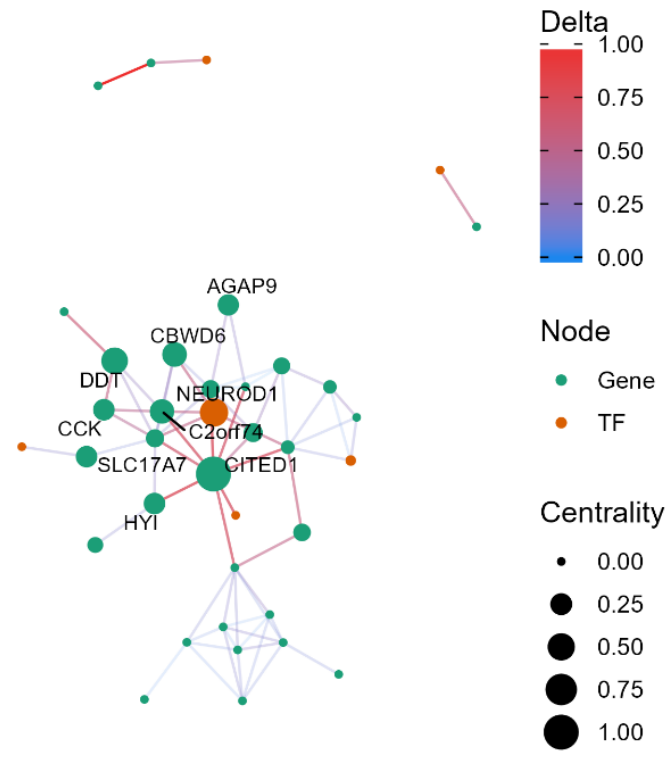

C

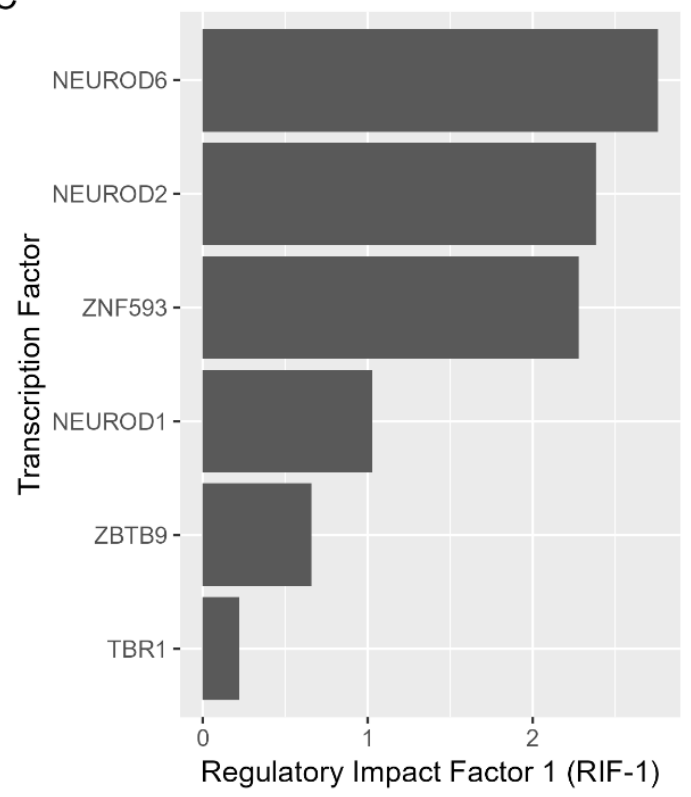

B

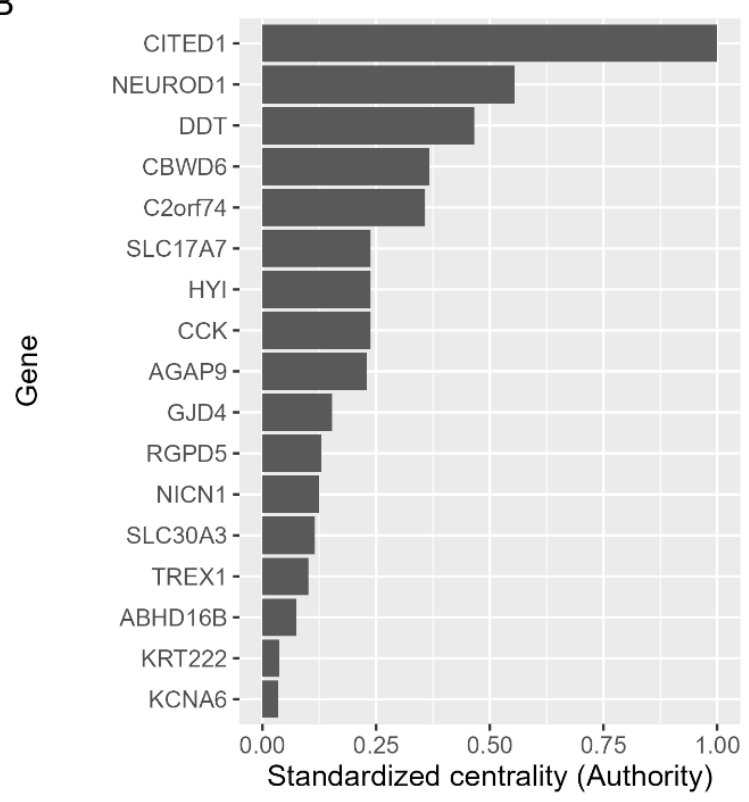

D

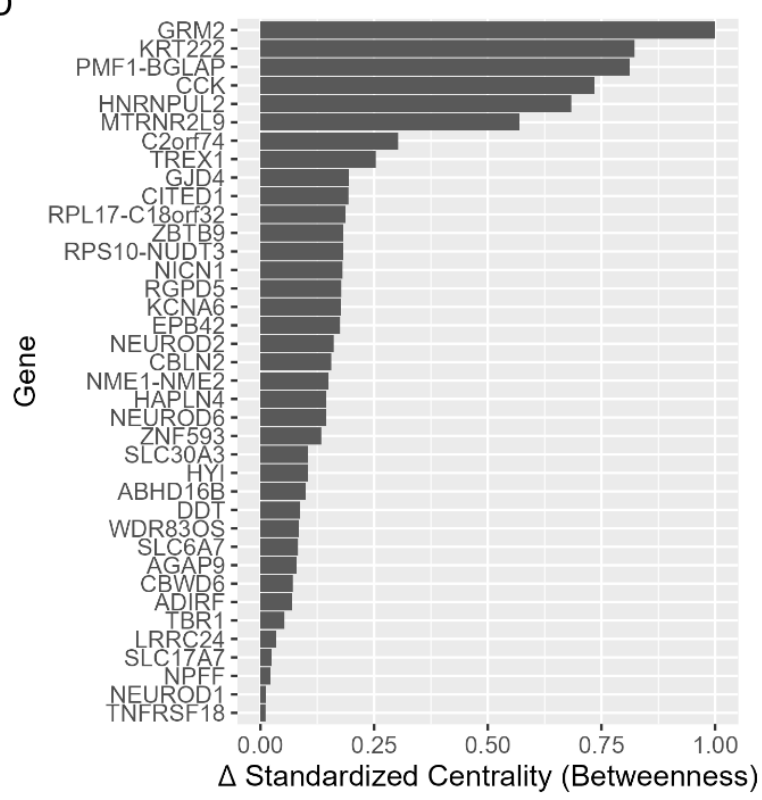

Figure 3. Differential network and measures of network regulators. (A) Differential network for PDC1 and PDC2 DEGs networks. (B) Bar plot for Standardized centrality of genes from the differential network. (C) RIF 1 Scores of genes from the differential network. D) Difference in standardized centrality between genes from PDC1 and PDC2 networks (shown in Figure 2B-C).

\subsection{Network regulators as drug repurposing candidates}

DEGs from PDC1 vs. PDC2 comparison were investigated to understand their role in the disease networks, aiming to identify potential drug repurposing candidate genes. CITED1 and NEUROD1 were identified as the most important genes regulating the differential wiring between clusters based on the authority centrality score (Figure 3B). The influence of the bHLH TFs (NEUROD1, NEUROD2, NEUROD6), along with ZNF593, was confirmed by RIF analysis, as these DEGs showed to be the best predictors of other DEGs expression levels in the disease network (Figure 3C). To further inspect the topology of the disease networks, we computed the delta standardized betweenness centrality score 
between PDC1 and PDC2, identifying GRM2, KRT222, PMF1-BGLPA, and CCK DEGs as the most differentially wired between the PDC1 and PDC2 clusters (Figure 3D). The obtained set of DEGs was used in the drug repurposing pipeline.

\subsection{Drug repurposing pipeline}

A drug repurposing analysis was performed to identify compounds modulating selected DEGs from the PDC1 vs. PDC2 comparison (table 1). In particular, we wanted to identify drugs showing agonist effects on the downregulated key genes driving the impaired neuroprotection network in PDC1. The obtained compounds $(n=42)$ were filtered and drugs being duplicated $(n=16)$ and inefficacious based on clinical trials $(n=5)$ were removed, along with substances showing side effects on memory or excitotoxicity $(n=2)$ and unknown modality of action $(n=10)$. The final set of drugs $(n=9)$ mapped on NEUROD1 and GRM2 genes. These nine compounds displayed different modalities of action that were assessed for both preclinical and clinical evidence of their neuroprotective potential (Table 1). 
Table 1. Compounds and drugs obtained from the drug repurposing pipeline. The Modality of action of each compound was identified on DGIdb and validated through extensive literature assessment, PMIDs are reported in the table.

\begin{tabular}{|c|c|c|c|c|c|}
\hline Gene & Drug & ChEMBL-ID & Phase & Modality of Action & PMID \\
\hline $\begin{array}{l}\text { NEU- } \\
\text { ROD1 }\end{array}$ & PD98059 & CHEMBL35482 & $\begin{array}{l}\text { Preclini- } \\
\text { cal }\end{array}$ & $\begin{array}{c}\text { ERK1/2 pathway inhibi- } \\
\text { tor }\end{array}$ & $\begin{array}{l}12297313 ; 28337120 ; \\
30274251 ; 16787571\end{array}$ \\
\hline $\begin{array}{l}\text { NEU- } \\
\text { ROD1 }\end{array}$ & $\begin{array}{l}\text { DEFEROX- } \\
\text { AMINE }\end{array}$ & CHEMBL556 & Launched & $\begin{array}{l}\text { hexadentate iron chela- } \\
\text { tor }\end{array}$ & $\begin{array}{c}16697980 ; 32926630 ; \\
23531432 ; 22754573 ; \\
31868679 ; 33513737 ; \\
33805195\end{array}$ \\
\hline $\begin{array}{l}\text { NEU- } \\
\text { ROD1 }\end{array}$ & ISX9 & CHEMBL1222381 & $\begin{array}{l}\text { Preclini- } \\
\quad \text { cal }\end{array}$ & $\begin{array}{l}\text { neural stem cell in- } \\
\text { ducer }\end{array}$ & $\begin{array}{l}29311646 ; 18552832 ; \\
26407349 ; 28656155 ; \\
22542682 ; 28216149\end{array}$ \\
\hline GRM2 & JNJ-40411813 & CHEMBL3337527 & Phase 2 & $\begin{array}{l}\text { glutamate receptor pos- } \\
\text { itive allosteric modula- } \\
\text { tor }\end{array}$ & $\begin{array}{c}25462291 ; 25586401 ; \\
25735992\end{array}$ \\
\hline GRM2 & LY2979165 & CHEMBL3544939 & Phase 2 & $\begin{array}{l}\text { glutamate receptor pos- } \\
\text { itive allosteric modula- } \\
\text { tor }\end{array}$ & $\begin{array}{c}32052375 ; 33071070 ; \\
29564482\end{array}$ \\
\hline GRM2 & LY2969822 & CHEMBL3545270 & Phase 1 & $\begin{array}{c}\text { glutamate receptor ag- } \\
\text { onist }\end{array}$ & $\begin{array}{c}28177520 ; 31306647 ; \\
30934533\end{array}$ \\
\hline GRM2 & LY404039 & CHEMBL375611 & Phase 1 & $\begin{array}{c}\text { glutamate receptor ag- } \\
\text { onist }\end{array}$ & $32403118 ; 32403118$ \\
\hline GRM2 & BINA & CHEMBL593013 & $\begin{array}{l}\text { Preclini- } \\
\text { cal }\end{array}$ & $\begin{array}{l}\text { glutamate receptor pos- } \\
\text { itive allosteric modula- } \\
\text { tor }\end{array}$ & $\begin{array}{c}16046122 ; 16608916 ; \\
17526600 ; 24076101 ; \\
28472649\end{array}$ \\
\hline GRM2 & CBiPES & CHEMBL4303163 & $\begin{array}{l}\text { Preclini- } \\
\text { cal }\end{array}$ & $\begin{array}{l}\text { glutamate receptor pos- } \\
\text { itive allosteric modula- } \\
\text { tor }\end{array}$ & $\begin{array}{c}15717213 ; 19951716 ; \\
22659090\end{array}$ \\
\hline
\end{tabular}

\subsection{Molecular characterization of PDC1 or PDC2 vs NC}

DEGs were identified for both PDC1 vs. NC and PDC2 vs. NC comparisons, aiming to assess the specific disease mechanisms of the two clusters (Figure 4). As expected, functional enrichment on PDMap enriched the entire diagram in the main map with p.adjusted $<0.0001$ for both clusters, including the Parkinsons UK Gene Ontology genes with p.adjusted $<0.05$ and p.adjusted $<0.0001$ for PDC1 and PDC2 respectively. Moreover, we studied the common DEGs resulting from each comparison to assess whether their connectivity was significantly different between PDC1 and PDC2. Two networks were built for both PDC1 and PDC2 common DEGs. Despite holding the same node structure, the networks were differentially connected (NCT, p < 0.0001), with 5000 differential connections showing p.adjusted (FDR) $<0.01$ (Figure S1). 


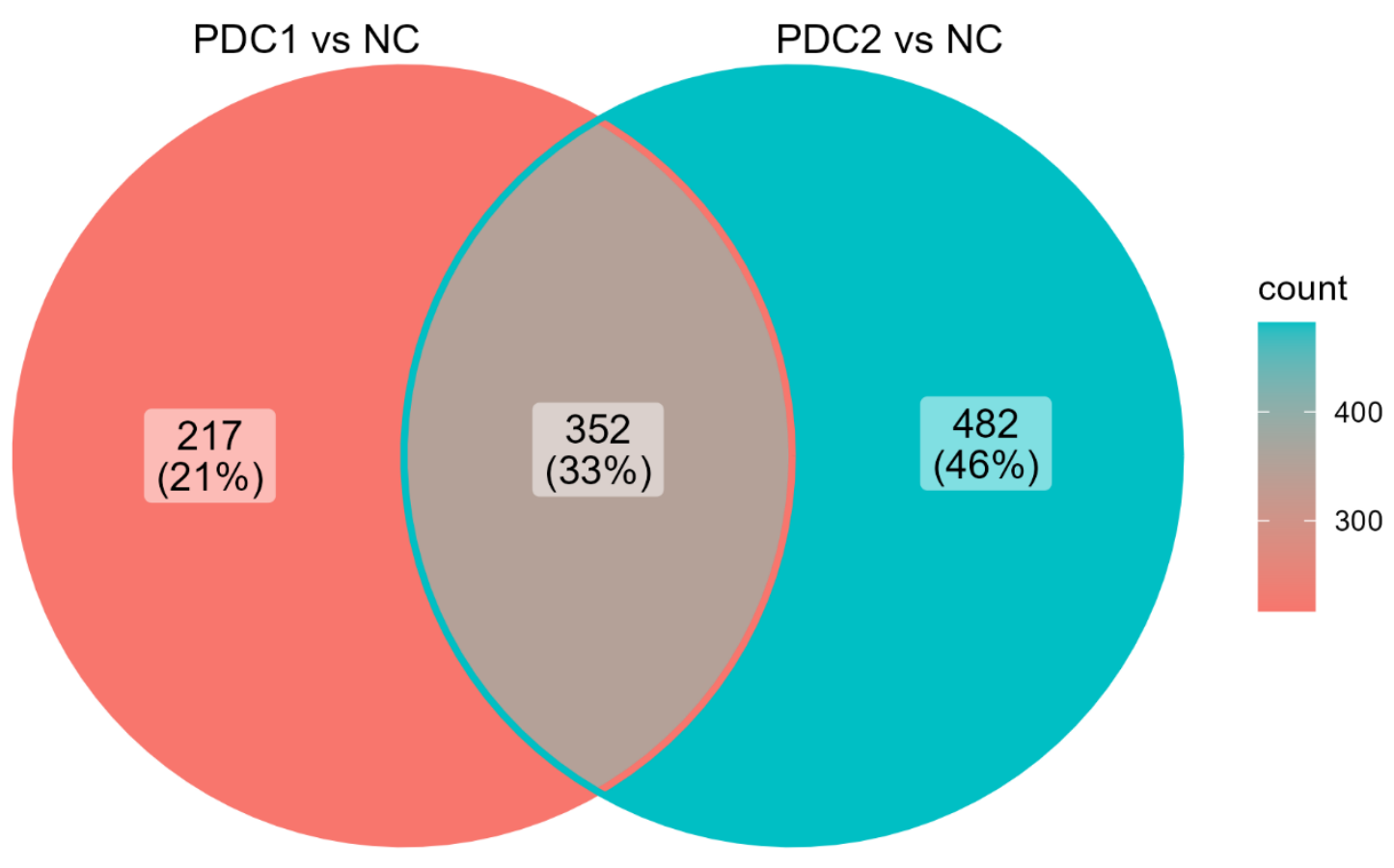

Figure 4. Venn diagram reporting the number of unique and overlapping DEGs found in PDC1 vs. NC and PDC2 vs. NC comparisons.

\subsubsection{Disease mechanism of PDC1}

217 unique DEGs were identified in the PDC1 vs. NC comparison with p.adjusted $($ FDR) $\leq 0.01$ (Figure 4). Within this set, 5 unique TFs were found and GDA analysis revealed that $12 \%$ of these DEGs were already associated with PD. Functional enrichment analysis mapped this set of DEGs on 15 biological pathways (p.adjusted < 0.05 ), mostly belonging to glutamatergic (KEGG:04724) and GABAergic signaling (CORUM:5418, REAC:R-HSA-991365, REAC:R-HSA-977444), but also including the Apelin signaling pathway (KEGG:04371) (Figure 5A) and the Sonic Hedgehog (SHH) pathway from the Parkinson's UK Annotation Initiative (p.adjusted $<0.05$ ) in the PDMap (Figure S2). The expression profiles of DEGs involved in the glutamatergic synapse were further detailed, highlighting the downregulation of several key genes underlying the glutamate balance in the synaptic cleft (Figure 5B) (GNG13, SLC17A7, GNG11, GRM2, SLC17A6, GRIK1, KCNJ3, SHANK1, GNG3). 
A

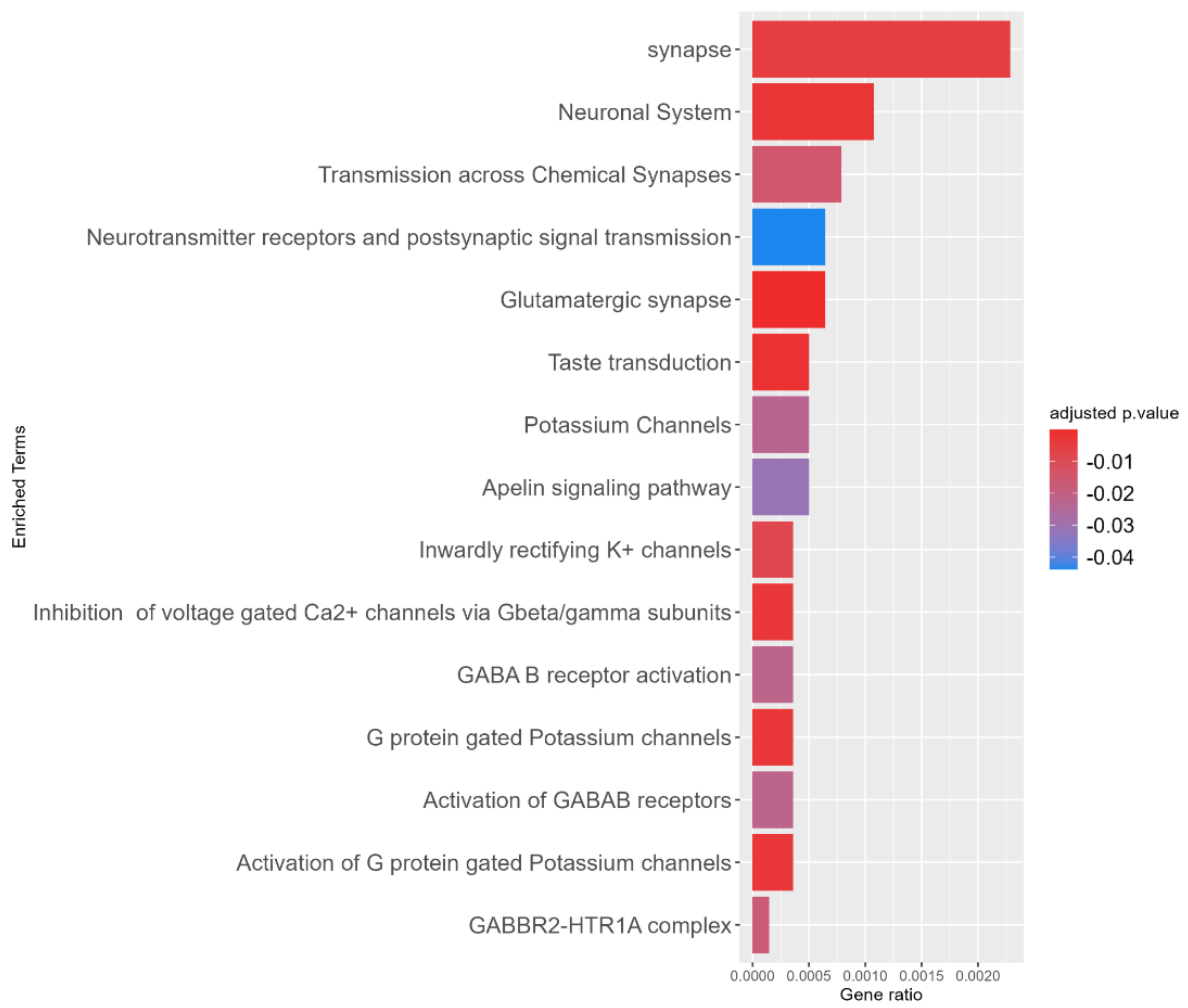

B

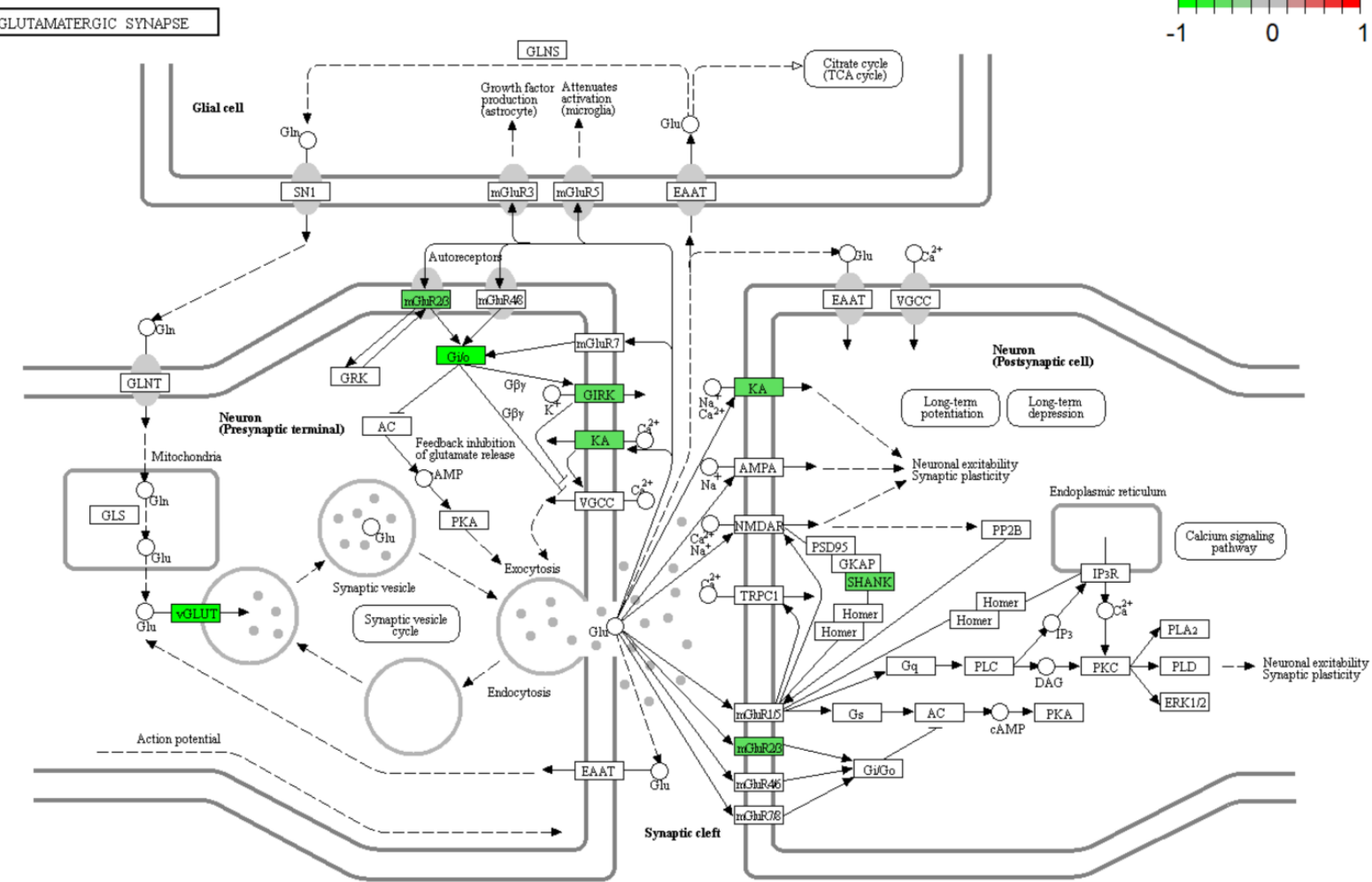

Figure 5. Investigation on the disease mechanism of PDC1, based on PDC1 vs. NC unique DEGs. (A) Enrichment analysis of DEGs shows that these genes map mostly on synaptic functions, in particular on the glutamatergic synapse. (B) Pheatmap illustration of the glutamatergic synapse pathway, showing downregulation in neurotransmitter uptake (vGLUT) and feedback regulation of neurotransmission (mGluR2) functions. 


\subsubsection{Disease mechanism of PDC2}

482 unique DEGs were identified in the PDC2 vs NC comparison, with p.adjusted $($ FDR) $\leq 0.01$ (Figure 4). Within this set, 2 unique TFs were found, while $10 \%$ of the DEGs were already associated with PD from GDA analysis. Functional enrichment analysis showed that PDC2 unique DEGs exclusively enriched pro-inflammatory pathways, namely Cell recruitment (pro-inflammatory response), Purinergic signaling in leishmaniasis infection, and Interleukin-1 processing from REACT database (Figure 6A), as well as the Endoplasmic Reticulum (ER) stress signaling (p.adjusted $<0.01$ ) and the Ubiquitin proteasome system (p.adjusted $<0.05$ ) from the Parkinson's UK Annotation Initiative in the PDMap (Figure S3). These pathways were enriched by the same set of genes: NFKB2, IL18, P2RX7, NFKB1, C3AR1, CASP1. A network analysis approach was used to further investigate this immunological subnetwork characterizing PDC2 (Figure 6B). Several communities of DEGs were found, and communities 1 and 4 reported significant PPI enrichment scores p.values (p.value $=0.002$; p.value $<0.0001$ ). Community 1 significantly enriched the cell surface receptor signaling pathway (p.value $<0.05$ ). STRINGdb text mining on this community suggested that this set of DEGs may be involved in neuronal apoptosis mediated by oxidative stress (CXCR4,4E-BP1, DR5, TNFRSF10B, TRAIL-R2, HSPB1, TNFRSF1A, BAG3) [23]. As expected, community 4 was mainly composed of genes from the immune system (adjusted p.value $=0.03$ ) 
A

Purinergic signaling in leishmaniasis infection -

है

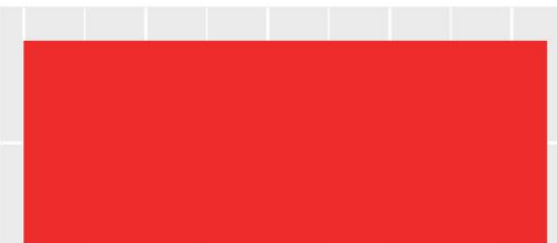

Cell recruitment (pro-inflammatory response) -

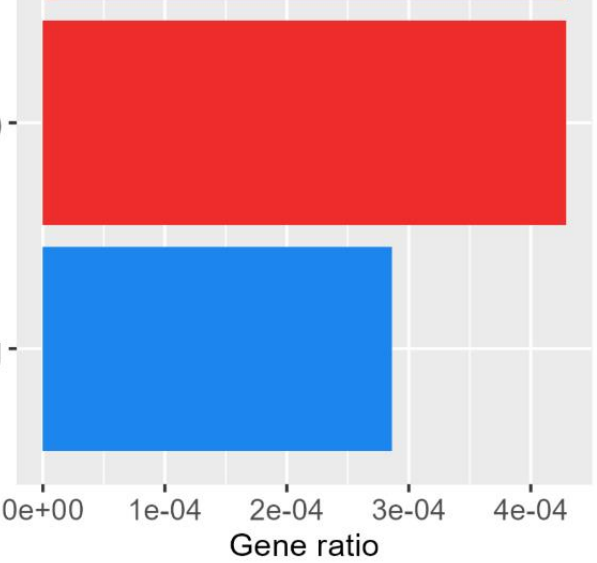

adjusted p.value

$-0.0150$

$-0.0151$

$-0.0152$

$-0.0153$

Interleukin-1 processing -

B

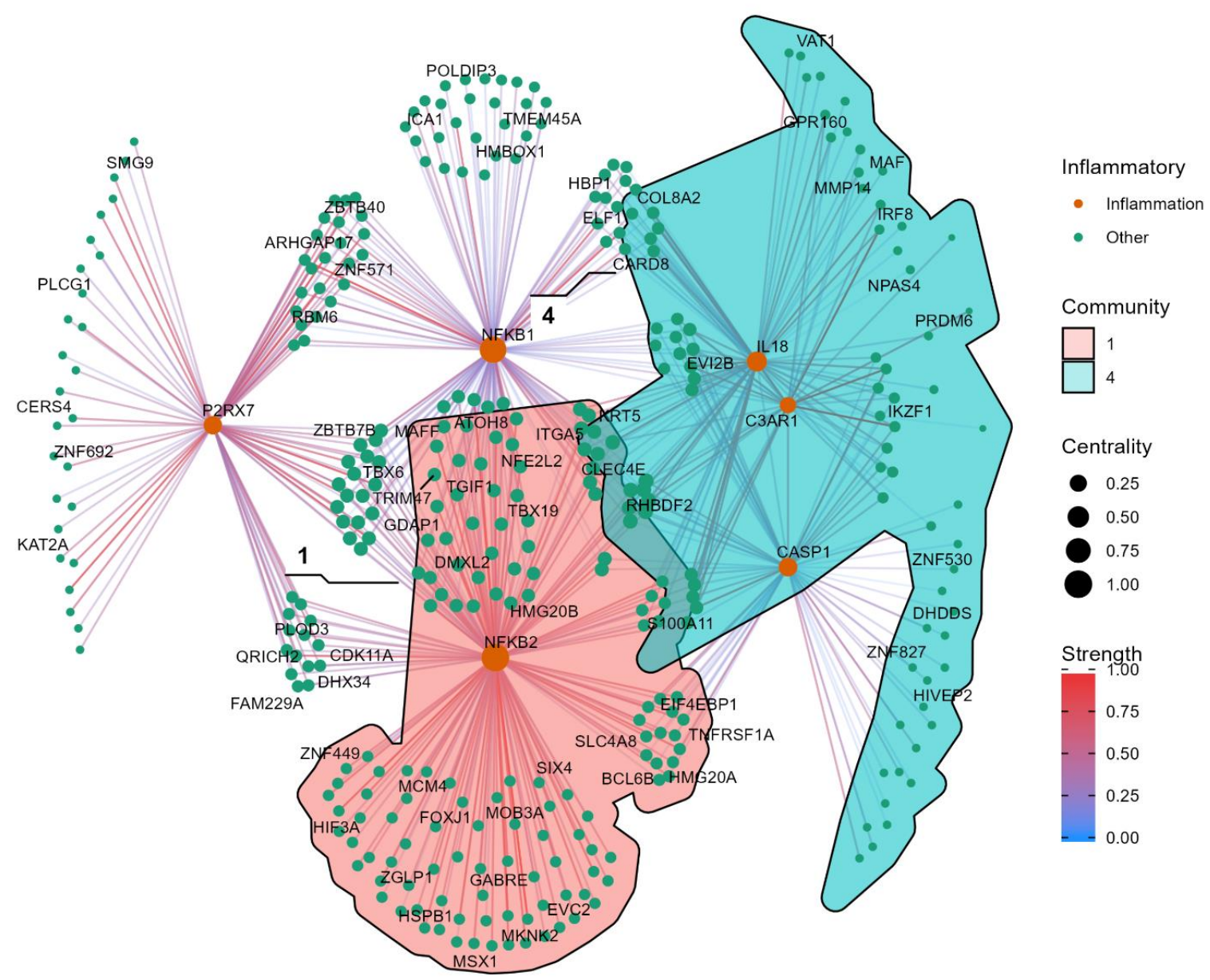

Figure 6. Investigation on the disease mechanism of PDC2, based on PDC2 vs. NC unique DEGs. (A) Enrichment analysis of DEGs shows that these genes map on inflammatory processes. (B) Gene network showing segregation in communities (only communities with significant PPI scores are colored). The network was built with the Kamada-Kawai layout, where each node is a gene and each edge is a PCIT value between genes. 


\section{Discussion}

Disease subtyping is required to address PD heterogeneity in clinical manifestations and progression and improve both management strategies and research in an attempt to develop disease-modifying treatments. In this study, we proposed a hybrid workflow integrating ML, bioinformatics and network analyses to overcome the pervasive limitations in PD subtyping, specifically addressing reproducibility, disease network identification and characterization, drug repurposing, and knowledge transferability. As a proof of concept, we analyzed RNA-Seq data from PD and NC post mortem brain samples to identify PD subtypes and to characterize the gene networks regulating common and specific disease mechanisms. In fact, we applied a data-driven approach based on network analysis and information theory and determined the most influential DEGs. Finally, we applied a drug repurposing pipeline to propose compounds holding therapeutic potential. Two PD subtypes were identified and internally validated using unsupervised ML. The obtained clusters differed by lifespan and Braak score, with PDC2 reporting a later age of death and lower neuronal depletion than PDC1. Additionally, we observed that PDC1 and PDC2 were characterized by specific disease mechanisms when compared with NC, further confirming that PDC1 and PDC2 represented two distinct subpopulations in PD. Alterations in SHH and Apelin signaling, coupled with altered glutamatergic transmission specifically characterized PDC1, while PDC2 unique disease fingerprint reported ER and oxidative stress mechanism due to the increase in neuroinflammation. Most of the differences between PDC1 and PDC2 gene expression were driven by a gene network centered on the bHLH NEUROD TFs (NEUROD1, NEUROD2, NEUROD6) controlling cell-cycle and adult neurogenesis in the Wnt $\beta$-catenin pathway $[24,25]$. Genes in this community were downregulated in PDC1 vs. PDC2, suggesting an impairment of Wnt $\beta$-catenin signaling. The impairment of Wnt/ $\beta$-catenin is a PD hallmark, as it regulates dopaminergic neurogenesis and survival in the subventricular zone and substantia nigra during aging [26]. Several other key genes in the Wnt/ $\beta$-catenin were downregulated in PDC1, including NFATc1 and CBP/p300 modulators (CITED1, eEF1B $\gamma$ ). CBP and p300 are epigenetic factors constituting the KAT3 family, which controls chromatin acetylation [27]. Differential $\mathrm{CBP} / \mathrm{p} 300$ modulation is used to regulate nuclear receptor/Wnt $/ \beta$-catenin interactions, allowing for both the maintenance of genomic integrity and neuronal plasticity during aging [28]. The present extensive network analysis revealed that NEUROD1 and GRM2 were the most influential genes in the network, as they were able to predict and/or influence the expression of the other DEGs. NEUROD1 regulates the onset of neurogenesis, differentiation, and survival in the Wnt/ $\beta$-catenin pathway [29-31], representing a key target of several applications aiming to restore dopaminergic signaling in PD [32-36]. In particular, NEUROD1 chemical reprogramming has produced dopaminergic neurons from astrocytes in an in vivo PD mouse model, while its overexpression reduced the loss of the dopaminergic neurons associated with $\mathrm{PD}$, thus inducing relief of symptoms $[22,24,37,38]$. GRM2 encodes mGluR2 metabotropic receptors modulating neurotransmission and synaptic plasticity between the substantia nigra and subthalamic nucleus [39]. Recently, GRM2 downregulation in substantia nigra has been associated with PD [40] since mGluR2 is essential for the induction of Long-Term Depression (LTD) in the substantia nigra and mGluR2 agonists have been proposed for the treatment of motor symptoms in PD [4143]. Given the therapeutic potential of NEUROD1 and GRM2, we used them in a drug repurposing pipeline, finding nine compounds potentially able to restore their functionality. NEUROD1-interacting compounds (ISX9, PD98059, Deferoxamine) are currently under evaluation as disease-modifying treatments in PD [4]. ISX9 is a neural stem cell inducer aiming to enhance adult neurogenesis through NEUROD1 overexpression [33,4446]. PD 98059 acts as an ERK1/2 inhibitor, counteracting the apoptotic processes harming dopaminergic neurons [45-48]. Finally, Deferoxamine (DFO) is an hexadentate iron chelator approved by the Food and Drugs Administration (FDA) to be prescribed for iron and aluminum intoxication [49]. Given that free iron and aluminum deposits are known to be involved in PD pathogenesis, iron chelation may represent a promising therapeutic strategy to improve behavioral outcomes and slow down neurodegeneration $[50,51]$. In 
PD animal models, DFO chronic intranasal administration reduces motor defects and overall pathology, while it has been demonstrated that DFO treatment increases the number of neurons produced from neural stem/progenitor cells (NPCs) due to the activation of the NEUROD signaling pathway [52,53]. DFO action can also be coupled with antioxidants to counteract neurotoxicity in dopaminergic neurons of the substantia nigra, reducing oxidative stress and cellular damage [54]. Clinical trials on an orally active form of the iron chelator deferiprone (DFP) showed decreases in substantia nigra iron content resulting in improved Unified Parkinson's Disease Rating Scale (UPDRS) scores [55]. GRM2 modulators have risen in interest, given that allosteric modulators of $G$ protein-coupled receptors (GPCRs) appear to provide a new strategy to develop novel treatments in neurodegenerative diseases in general and PD in particular [43]. Moreover, GPCRs Positive Allosteric Modulators (PAM) acting on mGluR2 can potentiate the receptor response providing higher subtype selectivity and thus reduce the activation of pathways inducing side-effects [56].

\section{Materials and methods}

\subsection{Systematic data retrieval}

A comprehensive online search of published literature in Gene Expression Omnibus (GEO) and PubMed databases (to April 30, 2021) was performed to identify all publications measuring RNA expression levels in brain tissue from idiopathic PD patients. The search strategy used a query string including as relevant keywords: "Parkinson, High throughput Sequencing". Studies were included to retrieve data if they met the following criteria: available RNA-Seq data; Sequenced RNA extracted from post-mortem human brain tissue; RNA sequenced by Next Generation Sequencing (NGS) technology; data must include both PD and Normal Control (NC) subjects' expression matrices. Search results were reviewed by two investigators (A.T. and C.F.) who were required to agree on study selection. Any discrepancies were resolved by discussion. Preferred Reporting Items for Systematic Reviews and Meta-Analyses (PRISMA) guidelines adherence was respected to perform this systematic data retrieval [59]. After filtering, 6 datasets including transcriptomic data of 210 participants ( $\mathrm{PD}=115 ; \mathrm{NC}=94$ ) were selected (Figure 7), namely GSE136666 [40], GSE135036 [57], GSE134390, GSE110716 [58], GSE68719 [59], and [60]. 


\section{Records identified through GEO $(n=48)$}

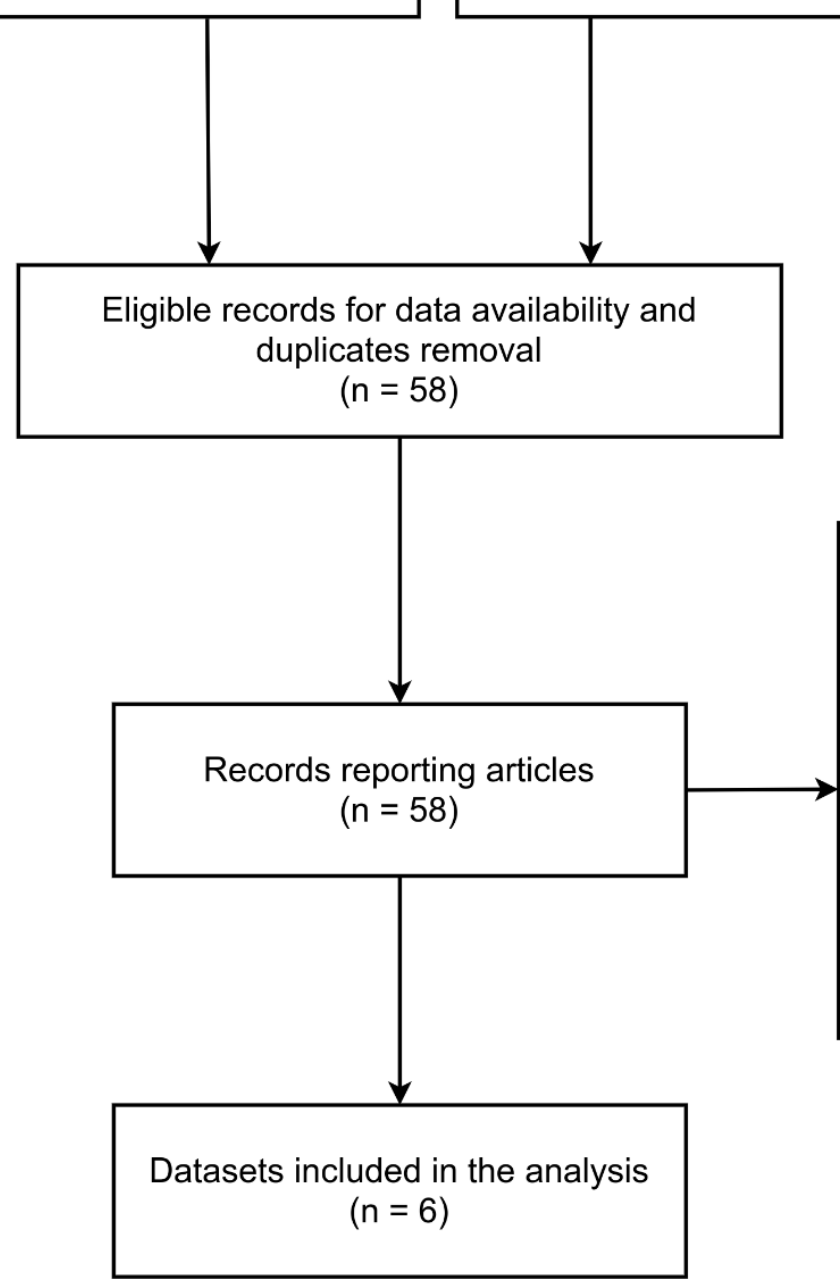

Records identified through PubMed $(n=107)$

\section{Records excluded}

$(n=52)$

- Animal studies $(n=17)$

- Expression profiling by array ( $\mathrm{n}$ = 29)

- Group bias $(n=2)$

- Corrections $(n=3)$

- Retraction notes $(n=1)$

Figure 7. PRISMA flow diagram showing the step-by-step process of our search and selection applied during systematic data retrieval.

\subsection{Raw counts preprocessing}

Raw counts matrices were integrated by gene, and univariate anomaly detection on demographics was performed using Grubbs Test. One PD subject that proved to be an outlier for the age of death parameter was then removed. Genes with expression values below the $15^{\text {th }}$ percentile were removed during the low counts filtering stage. The batch effect due to the experiment source was removed using the "ComBat-seq" function in the sva package [61,62]. "ComBat-seq" uses a negative binomial regression model to estimate batch effects based on the count matrix in RNA-seq studies. The estimated batch effect parameters are used to calculate the expected distributions if there were no batch effects in the data based on the model. Then, the "ComBat-seq" function adjusts the data by mapping the quantiles of the empirical distributions of data to the batch-free distributions [63]. Preprocessed gene counts matrix for the clustering pipeline were included in supplementary table 1. 


\subsection{Clustering pipeline}

After raw counts preprocessing, the clustering tendency of the PD gene-expression data was confirmed using Hopkins statistic $(\mathrm{H}=0.14)$, while the best number of clusters $(n=2)$ was estimated using the NbClust $\mathrm{R}$ package $[64,65]$. NbClust's approach to clustering validity is based on the relative criteria, which consists in the evaluation of a clustering structure by comparing it with other clustering schemes resulting from the same algorithm but with different parameter values [65]. The clustering pipeline included 7 algorithms from the factoextra $\mathrm{R}$ package [66](Fuzzy clustering; k-means, Hierarchical_kmeans; clara, agnes; pam; hclust; diana) over several distance metrics and measures (Supplementary Table 2) and the final clustering method was selected based on the maximum average Silhouette width and Dunn index methods (Supplementary Table 2). The silhouette (S) and Dunn (D) coefficients measure how similar an observation is to its own cluster (cohesion) compared to other clusters (separation), which indicates how well an observation was classified [67]. Since $S$ and D coefficients can be computed with any distance metric, they can be compared across multiple models. Moreover, it was demonstrated that $\mathrm{S}$ and $\mathrm{D}$ coefficients are among the most reliable internal validation metrics in five different aspects: monotonicity, noise, density, subclusters, and skewed distributions [68].

\subsection{Statistical analyses}

All the statistical analyses were performed in R v. 4.1.0 [69]. A seed was set to 12345 for all algorithms needing pseudorandomization. A Wilcoxon rank-sum test was performed to assess differences in age of death in PDC1 vs PDC2 comparison [70], whereas Fisher tests were used to evaluate differences in Braak score and sex in the same comparison [71].

\subsection{Differential expression analysis}

Differential expression analysis was performed using limma for each comparison and gene expression matrices were transformed in $\log _{2}$ counts per million (CPM) before modeling [72]. Finally, moderated t-statistic and F-statistic along with log-odds of differential expression were computed by empirical Bayes moderation of the standard errors. Only genes with $p$-value adjusted for False Discovery Rate (FDR) $\leq 0.01$ were considered Differentially Expressed Genes (DEGs) [73]. Over Representation Analysis (ORA) was performed to map genes on their functional pathways. DEGs were queried on all Gene Ontology (GO) domains: Biological Processes (BP), Cellular Components (CC) and Molecular Functions (MF). Similarly, DEGs were mapped on the CORUM database, which provides manually annotated protein complexes, and the Kyoto Encyclopedia of Genes and Genomes (KEGG) database using g:Profiler API [74]. Moreover, a Gene-Disease Association (GDA) analysis was performed using DisGeNET to retrieve DEGs associated with PD (tag:C0030567) [75]. Unique DEGS from PDC1 and PDC2 vs. NC comparisons were mapped on the Parkinson's Disease Map (PDMap), including the Parkinson's UK Annotation Initiative to identify their specific disease pathways [76]. The obtained pathways were considered as differentially modulated with p.adjusted $\leq 0.05$ (FDR) and KEGG pathways were visualized using the pathview package [77]. Critical TFs among DEGs were identified using the CeTF package, and Regulatory Impact Factors scores (RIF) were computed for each of the identified TFs [78]. Complete results from the differential expression analysis were reported in supplementary table 3 and supplementary table 4 for PDC1 vs. PDC2 and PDC1/2 vs NC respectively.

\subsection{Gene Network Analysis}

The gene networks in PDC1 and PDC2, as well as the differential network between the clusters, were investigated using the Partial Correlation coefficient with Information Theory (PCIT) algorithm to estimate connectivity among nodes. PCIT identifies meaningful correlations to define edges in a weighted gene co-expression network [78]. Importantly, PCIT allows one to draw a co-expression gene network while controlling the moderation effect of other genes. For example, for every trio of genes in $x, y$, and $z$, the partial correlation coefficient between $\mathrm{x}$ and $\mathrm{y}$ given $\mathrm{z}$ indicates the strength of the linear 
relationship between $\mathrm{x}$ and $\mathrm{y}$ that is independent of (uncorrelated with) z. Statistical significance of the association between $\mathrm{x}$ and $\mathrm{y}$ is evaluated by computing the tolerance level to be used as the local threshold for capturing significant associations for every trio of genes. The tolerance level here is defined as the average ratio of partial to direct correlation [79]. Finally, each network was optimized by pruning isolated nodes and looped or multiple edges. The obtained networks were represented using the force-directed Kamada-Kawai algorithm to assure that geometric distances between vertices closely corresponded to the underlying graph distances. Moreover, distinct communities of genes in PDC1 and PDC2 networks were identified using the label propagation algorithm from the tidygraph package, while weights of genes in the networks were estimated using the betweenness centrality score from the same package [80]. The functions of the identified communities and their Protein-Protein Interaction (PPI) enrichment scores were investigated using the Protein-Protein Interaction network from the STRINGdb package [81]. The networks were compared to identify both structural differences and changes in the overall level of connectivity using the NetworkComparisonTest (NCT) package [82]. To build the differential network in PDC1 vs. PDC2 comparison, we tested the edge strength invariance hypothesis, meaning that a specific edge is identical across subpopulations. The edges and nodes showing differential strength levels were used to represent the differential network between the two clusters, while the authority centrality measure was computed to estimate the weight of the nodes in the network [80].

\subsection{Drug repurposing}

The DEGs from PDC1 vs. PDC2 comparison were used in a drug repurposing pipeline. We selected as input the top-5 most influential DEGs in the gene network based on authority, RIF1, RIF2, and betweenness scores. The selected DEGs were queried on the Drug Gene Interaction Database (DGIdb) and the Drug Repurposing Hub from the Broad Institute to obtain their interacting compounds and modality of action. The Drug Repurposing Hub is a curated and annotated collection of FDA-approved drugs, clinical trial drugs, and pre-clinical tool compounds, while DGIdb is a data mining platform exploring the druggable genome for personalized medicine $[83,84]$. DGIdb mining algorithm explores over 30 trusted sources, including scientific literature and curated database [83]. The obtained set of drugs was filtered with the following criteria: duplicated, inefficacious, side effects on memory or excitotoxicity, and unknown modality of action. The final set of drugs was then evaluated based on their target and modality of action $[83,84]$. Each compound's literature data was filtered to include only preclinical experiments or concluded clinical trials on neurodegenerative diseases. Finally, data from ClinicalTrials.gov (accession date: 10/05/2021) were retrieved to verify ongoing clinical trials on neurodegenerative diseases involving the administration of the identified compounds [85].

\section{Conclusion}

Hybrid workflows combining network biology and artificial intelligence have the potential to discover novel mechanisms and promising drug targets for complex diseases [21]. Despite this potential, these techniques were rarely combined in a data-driven pipeline. We implemented a fully data-driven pipeline for PD subtyping, aiming to gather knowledge for precision medicine applications. Collectively, our results point out that two clusters representing distinct PD subpopulations can be identified from RNA-Seq data. Adopting a rigorous data-driven pipeline for disease subtyping allowed us to stratify homogeneous groups of individuals based on their unique pattern of gene expression. Interestingly, PD subjects belonging to PDC1 and PDC2 were consistently different in the $W n t / \beta$-Catenin signaling pathway, which regulates neuronal survival, adult neurogenesis, and plasticity. On the other hand, when compared to NC, PDC1 and PDC2 differed also by disease mechanisms. PDC1 was characterized by an altered glutamatergic transmission, while PDC2 was characterized by altered inflammatory pathways. These results were independently validated on the PDMAP from the University of Luxembourg, which is a manually curated knowledge repository established to describe molecular mechanisms of PD. These alterations in neuroprotection and distinct disease machinery were 
reflected on clinical data, as PDC1 and PDC2 were characterized by different ages at death and Braak scores. Using a data-driven methodology for subtypes identification and characterization could bring to the identification of disease-modifying treatments specifically suited for one subtype. To this extent, we performed a drug-repurposing pipeline using as input the key genes regulating the alteration in expression profiles of PDC1 and PDC2, identifying 9 drugs at various stages of FDA approval. This set of compounds shared a known or predicted neuroprotective effect. This workflow is in line with the aims of precision medicine, for which detecting disease subtypes enhances diagnostic precision, finally helping to determine specific treatments for well-defined patients [86]. Although researchers are using similar in silico approaches to unravel the regulatory pathways underneath complex diseases and to identify key druggable disease networks $[20,21,87]$, reduced data availability represents a major limitation in this workflow, as pivotal clinical information like age of PD onset, treatment type and duration, or comorbidities is often lacking in public repositories. The lack of clinical characteristics in in silico unsupervised analyses prevents this kind of application to be timely translated into clinical practice. Moreover, limitations in data availability hindered knowledge translation from the brain to more easily available tissues in clinical practice, such as blood samples. Thus, the translation of the findings into biological understanding remains a major challenge to be addressed in future research. As the field moves forward, better data practices are needed to keep up with the increasing availability of novel technologies and the need to implement artificial intelligence tools for biomedical purposes [88]. Here, we showed how transcriptomic data and unsupervised ML can be leveraged to identify and characterize distinct subpopulations of idiopathic PD while proposing tailored potential treatments, enabling a precision medicine approach to complex neurodegenerative diseases.

Supplementary Materials: The following are available online at https://www.mdpi.com/xxxxxxx

Author Contributions: conceptualization, A.T., C.F.; methodology, A.T., C.F., C.S., V.C.; software, A.T., C.F.; validation, all authors; formal analysis, A.T., C.F.; investigation, A.T., C.F.; resources, A.T., C.F., C.C., E.G.; data curation, A.T., C.F.; writing-original draft preparation, A.T., C.F.; writing-review and editing, all authors; visualization, A.T., C.F.; supervision, L.P., C.C., R.C., E.G.; project administration, C.C., R.C., E.G. All authors have read and agreed to the published version of the manuscript.

Funding: This research received no external funding.

Institutional Review Board Statement: Not applicable.

Informed Consent Statement: Not applicable.

Data Availability Statement: Original data is available on NCBI Gene Expression Omnibus at https://www.ncbi.nlm.nih.gov/geo/ . The preprocessed data table, ready for the unsupervised Machine Learning pipeline, is available in the supplementary data.

Acknowledgements: We thank Filippo Taranta for his support in data curation, and Luca Battistini and Giovanna Borsellino for their assistance and guidance.

Conflicts of Interest: The authors declare no conflict of interest

\section{References}

1. Bloem, B.R.; Okun, M.S.; Klein, C. Parkinson's Disease. The Lancet 2021, 397, 2284-2303, doi:10.1016/S01406736(21)00218-X.

2. Deuschl, G.; Beghi, E.; Fazekas, F.; Varga, T.; Christoforidi, K.A.; Sipido, E.; Bassetti, C.L.; Vos, T.; Feigin, V.L. The Burden of Neurological Diseases in Europe: An Analysis for the Global Burden of Disease Study 2017. Lancet Public Health 2020, 5, e551-e567, doi:10.1016/S2468-2667(20)30190-0.

3. Dorsey, E.R.; Elbaz, A.; Nichols, E.; Abd-Allah, F.; Abdelalim, A.; Adsuar, J.C.; Ansha, M.G.; Brayne, C.; Choi, J.-Y.J.; Collado-Mateo, D.; et al. Global, Regional, and National Burden of Parkinson's Disease, 1990-2016: A 
Systematic Analysis for the Global Burden of Disease Study 2016. Lancet Neurol. 2018, 17, 939-953, doi:10.1016/S1474-4422(18)30295-3.

4. Lang, A.E.; Espay, A.J. Disease Modification in Parkinson's Disease: Current Approaches, Challenges, and Future Considerations. Mov. Disord. Off. J. Mov. Disord. Soc. 2018, 33, 660-677, doi:10.1002/mds.27360.

5. Paolini Paoletti, F.; Gaetani, L.; Parnetti, L. The Challenge of Disease-Modifying Therapies in Parkinson's Disease: Role of CSF Biomarkers. Biomolecules 2020, 10, 335, doi:10.3390/biom10020335.

6. Park, A.; Stacy, M. Disease-Modifying Drugs in Parkinson's Disease. Drugs 2015, 75, 2065-2071, doi:10.1007/s40265-015-0497-4.

7. Blauwendraat, C.; Nalls, M.A.; Singleton, A.B. The Genetic Architecture of Parkinson's Disease. Lancet Neurol. 2020, 19, 170-178, doi:10.1016/S1474-4422(19)30287-X.

8. Nalls, M.A.; Blauwendraat, C.; Vallerga, C.L.; Heilbron, K.; Bandres-Ciga, S.; Chang, D.; Tan, M.; Kia, D.A.; Noyce, A.J.; Xue, A.; et al. Identification of Novel Risk Loci, Causal Insights, and Heritable Risk for Parkinson's Disease: A Meta-Analysis of Genome-Wide Association Studies. Lancet Neurol. 2019, 18, 1091-1102, doi:10.1016/S1474-4422(19)30320-5.

9. Chang, D.; Nalls, M.A.; Hallgrímsdóttir, I.B.; Hunkapiller, J.; van der Brug, M.; Cai, F.; International Parkinson's Disease Genomics Consortium; 23andMe Research Team; Kerchner, G.A.; Ayalon, G.; et al. A Meta-Analysis of Genome-Wide Association Studies Identifies 17 New Parkinson's Disease Risk Loci. Nat. Genet. 2017, 49, 1511-1516, doi:10.1038/ng.3955.

10. International Parkinson Disease Genomics Consortium; Nalls, M.A.; Plagnol, V.; Hernandez, D.G.; Sharma, M.; Sheerin, U.-M.; Saad, M.; Simón-Sánchez, J.; Schulte, C.; Lesage, S.; et al. Imputation of Sequence Variants for Identification of Genetic Risks for Parkinson's Disease: A Meta-Analysis of Genome-Wide Association Studies. Lancet Lond. Engl. 2011, 377, 641-649, doi:10.1016/S0140-6736(10)62345-8.

11. Fereshtehnejad, S.-M.; Romenets, S.R.; Anang, J.B.M.; Latreille, V.; Gagnon, J.-F.; Postuma, R.B. New Clinical Subtypes of Parkinson Disease and Their Longitudinal Progression: A Prospective Cohort Comparison With Other Phenotypes. JAMA Neurol. 2015, 72, 863-873, doi:10.1001/jamaneurol.2015.0703.

12. Greenland, J.C.; Williams-Gray, C.H.; Barker, R.A. The Clinical Heterogeneity of Parkinson's Disease and Its Therapeutic Implications. Eur. J. Neurosci. 2019, 49, 328-338, doi:10.1111/ejn.14094.

13. Riggare, S.; Hägglund, M. Precision Medicine in Parkinson's Disease - Exploring Patient-Initiated Self-Tracking. J. Park. Dis. 2018, 8, 441-446, doi:10.3233/JPD-181314.

14. Severson, K.A.; Chahine, L.M.; Smolensky, L.A.; Dhuliawala, M.; Frasier, M.; Ng, K.; Ghosh, S.; Hu, J. Discovery of Parkinson's Disease States and Disease Progression Modelling: A Longitudinal Data Study Using Machine Learning. Lancet Digit. Health 2021, 3, e555-e564, doi:10.1016/S2589-7500(21)00101-1.

15. Fabrizio, C.; Termine, A.; Caltagirone, C.; Sancesario, G. Artificial Intelligence for Alzheimer's Disease: Promise or Challenge? Diagnostics 2021, 11, 1473, doi:10.3390/diagnostics11081473.

16. Qian, E.; Huang, Y. Subtyping of Parkinson's Disease - Where Are We Up To? Aging Dis. 2019, 10, 1130-1139, doi:10.14336/AD.2019.0112.

17. Bertucci Filho, D.; Teive, H.A.G.; Werneck, L.C. Early-Onset Parkinson's Disease and Depression. Arq. Neuropsiquiatr. 2007, 65, 5-10, doi:10.1590/s0004-282x2007000100003.

18. Wickremaratchi, M.M.; Knipe, M.D.W.; Sastry, B.S.D.; Morgan, E.; Jones, A.; Salmon, R.; Weiser, R.; Moran, M.; Davies, D.; Ebenezer, L.; et al. The Motor Phenotype of Parkinson's Disease in Relation to Age at Onset. Mov. Disord. Off. J. Mov. Disord. Soc. 2011, 26, 457-463, doi:10.1002/mds.23469.

19. Dienstmann, R.; Vermeulen, L.; Guinney, J.; Kopetz, S.; Tejpar, S.; Tabernero, J. Consensus Molecular Subtypes and the Evolution of Precision Medicine in Colorectal Cancer. Nat. Rev. Cancer 2017, 17, 79-92, doi:10.1038/nrc.2016.126.

20. Neff, R.A.; Wang, M.; Vatansever, S.; Guo, L.; Ming, C.; Wang, Q.; Wang, E.; Horgusluoglu-Moloch, E.; Song, W.; Li, A. Molecular Subtyping of Alzheimer's Disease Using RNA Sequencing Data Reveals Novel Mechanisms and Targets. Sci. Adv. 2021, 7, eabb5398.

21. Wang, Q.; Zhang, Y.; Wang, M.; Song, W.-M.; Shen, Q.; McKenzie, A.; Choi, I.; Zhou, X.; Pan, P.-Y.; Yue, Z.; et al. The Landscape of Multiscale Transcriptomic Networks and Key Regulators in Parkinson's Disease. Nat. Commun. 2019, 10, 5234, doi:10.1038/s41467-019-13144-y.

22. Ma, N.-X.; Yin, J.-C.; Chen, G. Transcriptome Analysis of Small Molecule-Mediated Astrocyte-to-Neuron Reprogramming. Front. Cell Dev. Biol. 2019, 7, 82, doi:10.3389/fcell.2019.00082.

23. Galluzzi, L.; Vitale, I.; Aaronson, S.A.; Abrams, J.M.; Adam, D.; Agostinis, P.; Alnemri, E.S.; Altucci, L.; Amelio, I.; Andrews, D.W.; et al. Molecular Mechanisms of Cell Death: Recommendations of the Nomenclature Committee on Cell Death 2018. Cell Death Differ. 2018, 25, 486-541, doi:10.1038/s41418-017-0012-4.

24. Tutukova, S.; Tarabykin, V.; Hernandez-Miranda, L.R. The Role of Neurod Genes in Brain Development, Function, and Disease. Front. Mol. Neurosci. 2021, 14, 109, doi:10.3389/fnmol.2021.662774.

25. Marchetti, B. Wnt/ $\beta$-Catenin Signaling Pathway Governs a Full Program for Dopaminergic Neuron Survival, Neurorescue and Regeneration in the MPTP Mouse Model of Parkinson's Disease. Int. J. Mol. Sci. 2018, 19, E3743, doi:10.3390/ijms19123743. 
26. Marchetti, B.; Tirolo, C.; L'Episcopo, F.; Caniglia, S.; Testa, N.; Smith, J.A.; Pluchino, S.; Serapide, M.F. Parkinson's Disease, Aging and Adult Neurogenesis: Wnt/ $\beta$-Catenin Signalling as the Key to Unlock the Mystery of Endogenous Brain Repair. Aging Cell 2020, 19, e13101, doi:10.1111/acel.13101.

27. Valor, L.M.; Viosca, J.; Lopez-Atalaya, J.P.; Barco, A. Lysine Acetyltransferases CBP and P300 as Therapeutic Targets in Cognitive and Neurodegenerative Disorders. Curr. Pharm. Des. 2013, 19, 5051-5064, doi:10.2174/13816128113199990382.

28. Ono, M.; Lai, K.K.Y.; Wu, K.; Nguyen, C.; Lin, D.P.; Murali, R.; Kahn, M. Nuclear Receptor/Wnt Beta-Catenin Interactions Are Regulated via Differential CBP/P300 Coactivator Usage. PloS One 2018, 13, e0200714, doi:10.1371/journal.pone.0200714.

29. Fu, M.; Wang, C.; Li, Z.; Sakamaki, T.; Pestell, R.G. Minireview: Cyclin D1: Normal and Abnormal Functions. Endocrinology 2004, 145, 5439-5447, doi:10.1210/en.2004-0959.

30. Hagey, D.W.; Topcic, D.; Kee, N.; Reynaud, F.; Bergsland, M.; Perlmann, T.; Muhr, J. CYCLIN-B1/2 and -D1 Act in Opposition to Coordinate Cortical Progenitor Self-Renewal and Lineage Commitment. Nat. Commun. 2020, 11, 2898, doi:10.1038/s41467-020-16597-8.

31. Xu, D.; Hou, K.; Li, F.; Chen, S.; Fang, W.; Li, Y. XQ-1H Alleviates Cerebral Ischemia in Mice through Inhibition of Apoptosis and Promotion of Neurogenesis in a Wnt/ $\beta$-Catenin Signaling Dependent Way. Life Sci. 2019, 235, 116844, doi:10.1016/j.lfs.2019.116844.

32. Brulet, R.; Matsuda, T.; Zhang, L.; Miranda, C.; Giacca, M.; Kaspar, B.K.; Nakashima, K.; Hsieh, J. NEUROD1 Instructs Neuronal Conversion in Non-Reactive Astrocytes. Stem Cell Rep. 2017, 8, 1506-1515, doi:10.1016/j.stemcr.2017.04.013.

33. Chang, J.-H.; Tsai, P.-H.; Wang, K.-Y.; Wei, Y.-T.; Chiou, S.-H.; Mou, C.-Y. Generation of Functional Dopaminergic Neurons from Reprogramming Fibroblasts by Nonviral-Based Mesoporous Silica Nanoparticles. Sci. Rep. 2018, 8, 11, doi:10.1038/s41598-017-18324-8.

34. Jiang, J.; Piao, X.; Hu, S.; Gao, J.; Bao, M. LncRNA H19 Diminishes Dopaminergic Neuron Loss by Mediating MicroRNA-301b-3p in Parkinson's Disease via the HPRT1-Mediated Wnt/ $\beta$-Catenin Signaling Pathway. Aging 2020, 12, 8820-8836, doi:10.18632/aging.102877.

35. Parmar, M.; Björklund, A.; Björklund, T. In Vivo Conversion of Dopamine Neurons in Mouse Models of Parkinson's Disease - a Future Approach for Regenerative Therapy? Curr. Opin. Genet. Dev. 2021, 70, 76-82, doi:10.1016/j.gde.2021.06.002.

36. Rivetti di Val Cervo, P.; Romanov, R.A.; Spigolon, G.; Masini, D.; Martín-Montañez, E.; Toledo, E.M.; La Manno, G.; Feyder, M.; Pifl, C.; Ng, Y.-H.; et al. Induction of Functional Dopamine Neurons from Human Astrocytes in Vitro and Mouse Astrocytes in a Parkinson's Disease Model. Nat. Biotechnol. 2017, 35, 444-452, doi:10.1038/nbt.3835.

37. Gao, Z.; Ure, K.; Ables, J.L.; Lagace, D.C.; Nave, K.-A.; Goebbels, S.; Eisch, A.J.; Hsieh, J. Neurod1 Is Essential for the Survival and Maturation of Adult-Born Neurons. Nat. Neurosci. 2009, 12, 1090-1092, doi:10.1038/nn.2385.

38. Pataskar, A.; Jung, J.; Smialowski, P.; Noack, F.; Calegari, F.; Straub, T.; Tiwari, V.K. NeuroD1 Reprograms Chromatin and Transcription Factor Landscapes to Induce the Neuronal Program. EMBO J. 2016, 35, 24-45, doi:10.15252/embj.201591206.

39. Dickerson, J.W.; Conn, P.J. Therapeutic Potential of Targeting Metabotropic Glutamate Receptors for Parkinson's Disease. Neurodegener. Dis. Manag. 2012, 2, 221-232, doi:10.2217/nmt.12.6.

40. Xicoy, H.; Brouwers, J.F.; Wieringa, B.; Martens, G.J.M. Explorative Combined Lipid and Transcriptomic Profiling of Substantia Nigra and Putamen in Parkinson's Disease. Cells 2020, 9, E1966, doi:10.3390/cells9091966.

41. Crupi, R.; Impellizzeri, D.; Cuzzocrea, S. Role of Metabotropic Glutamate Receptors in Neurological Disorders. Front. Mol. Neurosci. 2019, 12, 20, doi:10.3389/fnmol.2019.00020.

42. Murray, T.K.; Messenger, M.J.; Ward, M.A.; Woodhouse, S.; Osborne, D.J.; Duty, S.; O'Neill, M.J. Evaluation of the MGluR2/3 Agonist LY379268 in Rodent Models of Parkinson's Disease. Pharmacol. Biochem. Behav. 2002, 73, 455-466, doi:10.1016/s0091-3057(02)00842-0.

43. Nickols, H.H.; Conn, P.J. Development of Allosteric Modulators of GPCRs for Treatment of CNS Disorders. Neurobiol. Dis. 2014, 61, 10.1016/j.nbd.2013.09.013, doi:10.1016/j.nbd.2013.09.013.

44. Bettio, L.E.B.; Gil-Mohapel, J.; Patten, A.R.; O’Rourke, N.F.; Hanley, R.P.; Gopalakrishnan, K.; Wulff, J.E.; Christie, B.R. Effects of Isx-9 and Stress on Adult Hippocampal Neurogenesis: Experimental Considerations and Future Perspectives. Neurogenesis 2017, 4, e1317692, doi:10.1080/23262133.2017.1317692.

45. Petersen, H.V.; Jensen, J.N.; Stein, R.; Serup, P. Glucose Induced MAPK Signalling Influences NeuroD1-Mediated Activation and Nuclear Localization. FEBS Lett. 2002, 528, 241-245, doi:10.1016/s0014-5793(02)03318-5.

46. Rui, Y.; Sun, Z.; Gu, J.; Sheng, Z.; He, X.; Xie, Z. MEK Inhibitor PD98059 Acutely Inhibits Synchronized Spontaneous Ca2+ Oscillations in Cultured Hippocampal Networks. Acta Pharmacol. Sin. 2006, 27, 869-876, doi:10.1111/j.1745-7254.2006.00387.x.

47. Bohush, A.; Niewiadomska, G.; Filipek, A. Role of Mitogen Activated Protein Kinase Signaling in Parkinson's Disease. Int. J. Mol. Sci. 2018, 19, 2973, doi:10.3390/ijms19102973. 
48. Chen, G.; Nie, S.; Han, C.; Ma, K.; Xu, Y.; Zhang, Z.; Papa, S.M.; Cao, X. Antidyskinetic Effects of MEK Inhibitor Are Associated with Multiple Neurochemical Alterations in the Striatum of Hemiparkinsonian Rats. Front. Neurosci. 2017, 11, 112, doi:10.3389/fnins.2017.00112.

49. Kosyakovsky, J.; Fine, J.M.; Frey, W.H.; Hanson, L.R. Mechanisms of Intranasal Deferoxamine in Neurodegenerative and Neurovascular Disease. Pharm. Basel Switz. 2021, 14, 95, doi:10.3390/ph14020095.

50. Farr, A.C.; Xiong, M.P. Challenges and Opportunities of Deferoxamine Delivery for Treatment of Alzheimer's Disease, Parkinson's Disease, and Intracerebral Hemorrhage. Mol. Pharm. 2021, 18, 593-609, doi:10.1021/acs.molpharmaceut.0c00474.

51. Mounsey, R.B.; Teismann, P. Chelators in the Treatment of Iron Accumulation in Parkinson's Disease. Int. J. Cell Biol. 2012, 2012, e983245, doi:10.1155/2012/983245.

52. Febbraro, F.; Andersen, K.J.; Sanchez-Guajardo, V.; Tentillier, N.; Romero-Ramos, M. Chronic Intranasal Deferoxamine Ameliorates Motor Defects and Pathology in the $\alpha$-Synuclein RAAV Parkinson's Model. Exp. Neurol. 2013, 247, 45-58, doi:10.1016/j.expneurol.2013.03.017.

53. Kim, H.-J.; Hida, H.; Jung, C.-G.; Miura, Y.; Nishino, H. Treatment with Deferoxamine Increases Neurons from Neural Stem/Progenitor Cells. Brain Res. 2006, 1092, 1-15, doi:10.1016/j.brainres.2006.02.046.

54. Mursaleen, L.; Somavarapu, S.; Zariwala, M.G. Deferoxamine and Curcumin Loaded Nanocarriers Protect Against Rotenone-Induced Neurotoxicity. J. Park. Dis. 2020, 10, 99-111, doi:10.3233/JPD-191754.

55. Ward, R.J.; Dexter, D.T.; Martin-Bastida, A.; Crichton, R.R. Is Chelation Therapy a Potential Treatment for Parkinson's Disease? Int. J. Mol. Sci. 2021, 22, 3338, doi:10.3390/ijms22073338.

56. Foster, D.J.; Conn, P.J. Allosteric Modulation of GPCRs: New Insights and Potential Utility for Treatment of Schizophrenia and Other CNS Disorders. Neuron 2017, 94, 431-446, doi:10.1016/j.neuron.2017.03.016.

57. Li, P.; Ensink, E.; Lang, S.; Marshall, L.; Schilthuis, M.; Lamp, J.; Vega, I.; Labrie, V. Hemispheric Asymmetry in the Human Brain and in Parkinson's Disease Is Linked to Divergent Epigenetic Patterns in Neurons. Genome Biol. 2020, 21, 61, doi:10.1186/s13059-020-01960-1.

58. Schulze, M.; Sommer, A.; Plötz, S.; Farrell, M.; Winner, B.; Grosch, J.; Winkler, J.; Riemenschneider, M.J. Sporadic Parkinson's Disease Derived Neuronal Cells Show Disease-Specific MRNA and Small RNA Signatures with Abundant Deregulation of PiRNAs. Acta Neuropathol. Commun. 2018, 6, 58, doi:10.1186/s40478-018-0561$x$.

59. Dumitriu, A.; Golji, J.; Labadorf, A.T.; Gao, B.; Beach, T.G.; Myers, R.H.; Longo, K.A.; Latourelle, J.C. Integrative Analyses of Proteomics and RNA Transcriptomics Implicate Mitochondrial Processes, Protein Folding Pathways and GWAS Loci in Parkinson Disease. BMC Med. Genomics 2016, 9, 5, doi:10.1186/s12920-016-0164-y.

60. Nido, G.S.; Dick, F.; Toker, L.; Petersen, K.; Alves, G.; Tysnes, O.-B.; Jonassen, I.; Haugarvoll, K.; Tzoulis, C. Common Gene Expression Signatures in Parkinson's Disease Are Driven by Changes in Cell Composition. Acta Neuropathol. Commun. 2020, 8, 55, doi:10.1186/s40478-020-00932-7.

61. Leek, J.T. Svaseq: Removing Batch Effects and Other Unwanted Noise from Sequencing Data. Nucleic Acids Res. 2014, 42, e161-e161.

62. Leek, J.T.; Johnson, W.E.; Parker, H.S.; Jaffe, A.E.; Storey, J.D. The Sva Package for Removing Batch Effects and Other Unwanted Variation in High-Throughput Experiments. Bioinformatics 2012, 28, 882-883, doi:10.1093/bioinformatics/bts034.

63. Zhang, Y.; Parmigiani, G.; Johnson, W.E. ComBat-Seq: Batch Effect Adjustment for RNA-Seq Count Data. NAR Genomics Bioinforma. 2020, 2, lqaa078, doi:10.1093/nargab/lqaa078.

64. Banerjee, A.; Dave, R.N. Validating Clusters Using the Hopkins Statistic. In Proceedings of the 2004 IEEE International Conference on Fuzzy Systems (IEEE Cat. No.04CH37542); July 2004; Vol. 1, pp. 149-153 vol.1.

65. Charrad, M.; Ghazzali, N.; Boiteau, V.; Niknafs, A. NbClust: An R Package for Determining the Relevant Number of Clusters in a Data Set. J. Stat. Softw. 2014, 61, 1-36, doi:10.18637/jss.v061.i06.

66. Kassambara, A.; Mundt, F. Package 'Factoextra.' Extr. Vis. Results Multivar. Data Anal. 2017, 76.

67. Rousseeuw, P.J. Silhouettes: A Graphical Aid to the Interpretation and Validation of Cluster Analysis. J. Comput. Appl. Math. 1987, 20, 53-65.

68. Liu, Y.; Li, Z.; Xiong, H.; Gao, X.; Wu, J. Understanding of Internal Clustering Validation Measures. In Proceedings of the 2010 IEEE international conference on data mining; IEEE, 2010; pp. 911-916.

69. R Core Team R: A Language and Environment for Statistical Computing. 2013.

70. Hollander, M.; Wolfe, D.A.; Chicken, E. Nonparametric Statistical Methods; John Wiley \& Sons, 2013; Vol. 751; ISBN 1-118-55329-2.

71. Agresti, A. An Introduction to Categorical Data Analysis; John Wiley \& Sons, 2018; ISBN 1-119-40526-2.

72. Smyth, G.K. Limma: Linear Models for Microarray Data. In Bioinformatics and computational biology solutions using $R$ and Bioconductor; Springer, 2005; pp. 397-420.

73. Benjamini, Y. Discovering the False Discovery Rate. J. R. Stat. Soc. Ser. B Stat. Methodol. 2010, 72, 405-416. 
74. Raudvere, U.; Kolberg, L.; Kuzmin, I.; Arak, T.; Adler, P.; Peterson, H.; Vilo, J. G:Profiler: A Web Server for Functional Enrichment Analysis and Conversions of Gene Lists (2019 Update). Nucleic Acids Res. 2019, 47, W191-W198, doi:10.1093/nar/gkz369.

75. Piñero, J.; Ramírez-Anguita, J.M.; Saüch-Pitarch, J.; Ronzano, F.; Centeno, E.; Sanz, F.; Furlong, L.I. The DisGeNET Knowledge Platform for Disease Genomics: 2019 Update. Nucleic Acids Res. 2020, 48, D845-D855.

76. Fujita, K.A.; Ostaszewski, M.; Matsuoka, Y.; Ghosh, S.; Glaab, E.; Trefois, C.; Crespo, I.; Perumal, T.M.; Jurkowski, W.; Antony, P.M.A.; et al. Integrating Pathways of Parkinson's Disease in a Molecular Interaction Map. Mol. Neurobiol. 2014, 49, 88-102, doi:10.1007/s12035-013-8489-4.

77. Luo, W.; Brouwer, C. Pathview: An R/Bioconductor Package for Pathway-Based Data Integration and Visualization. Bioinformatics 2013, 29, 1830-1831.

78. Oliveira de Biagi, C.A.; Nociti, R.P.; Brotto, D.B.; Funicheli, B.O.; Cássia Ruy, P. de; Bianchi Ximenez, J.P.; Alves Figueiredo, D.L.; Araújo Silva, W. CeTF: An R/Bioconductor Package for Transcription Factor Co-Expression Networks Using Regulatory Impact Factors (RIF) and Partial Correlation and Information (PCIT) Analysis. BMC Genomics 2021, 22, 1-8.

79. Reverter, A.; Hudson, N.J.; Nagaraj, S.H.; Pérez-Enciso, M.; Dalrymple, B.P. Regulatory Impact Factors: Unraveling the Transcriptional Regulation of Complex Traits from Expression Data. Bioinformatics 2010, 26, 896-904, doi:10.1093/bioinformatics/btq051.

80. Pedersen, T.L. Tidygraph: A Tidy API for Graph Manipulation; 2020;

81. Szklarczyk, D.; Gable, A.L.; Lyon, D.; Junge, A.; Wyder, S.; Huerta-Cepas, J.; Simonovic, M.; Doncheva, N.T.; Morris, J.H.; Bork, P.; et al. STRING V11: Protein-Protein Association Networks with Increased Coverage, Supporting Functional Discovery in Genome-Wide Experimental Datasets. Nucleic Acids Res. 2019, 47, D607-D613, doi:10.1093/nar/gky1131.

82. Van Borkulo, C.D.; Boschloo, L.; Kossakowski, J.; Tio, P.; Schoevers, R.A.; Borsboom, D.; Waldorp, L.J. Comparing Network Structures on Three Aspects: A Permutation Test. J. Stat. Softw. 2017, 10.

83. Corsello, S.M.; Bittker, J.A.; Liu, Z.; Gould, J.; McCarren, P.; Hirschman, J.E.; Johnston, S.E.; Vrcic, A.; Wong, B.; Khan, M.; et al. The Drug Repurposing Hub: A next-Generation Drug Library and Information Resource. Nat. Med. 2017, 23, 405-408, doi:10.1038/nm.4306.

84. Freshour, S.L.; Kiwala, S.; Cotto, K.C.; Coffman, A.C.; McMichael, J.F.; Song, J.J.; Griffith, M.; Griffith, O.L.; Wagner, A.H. Integration of the Drug-Gene Interaction Database (DGIdb 4.0) with Open Crowdsource Efforts. Nucleic Acids Res. 2021, 49, D1144-D1151, doi:10.1093/nar/gkaa1084.

85. Zarin, D.A.; Tse, T.; Williams, R.J.; Califf, R.M.; Ide, N.C. The ClinicalTrials.Gov Results Database - Update and Key Issues. N. Engl. J. Med. 2011, 364, 852-860, doi:10.1056/NEJMsa1012065.

86. National Research Council; Studies, D. on E. and L.; Sciences, B. on L.; Disease, C. on A.F. for D. a N.T. of Toward Precision Medicine: Building a Knowledge Network for Biomedical Research and a New Taxonomy of Disease; National Academies Press, 2011; ISBN 978-0-309-22225-9.

87. Strafella, C.; Caputo, V.; Termine, A.; Fabrizio, C.; Ruffo, P.; Potenza, S.; Cusumano, A.; Ricci, F.; Caltagirone, C.; Giardina, E. Genetic Determinants Highlight the Existence of Shared Etiopathogenetic Mechanisms Characterizing Age-Related Macular Degeneration and Neurodegenerative Disorders. Front. Neurol. $2021,12$.

88. Shamseer, L.; Moher, D.; Clarke, M.; Ghersi, D.; Liberati, A.; Petticrew, M.; Shekelle, P.; Stewart, L.A. Preferred Reporting Items for Systematic Review and Meta-Analysis Protocols (PRISMA-P) 2015: Elaboration and Explanation. Bmj 2015, 349. 